J. Korean Math. Soc. 43 (2006), No. 5, pp. 991-1018

\title{
A THEORY OF RESTRICTED REGULARITY OF HYPERMAPS
}

\author{
Antonio Breda D'Azevedo
}

\begin{abstract}
Hypermaps are cellular embeddings of hypergraphs in compact and connected surfaces, and are a generalisation of maps, that is, 2-cellular decompositions of closed surfaces. There is a well known correspondence between hypermaps and co-compact subgroups of the free product $\Delta=C_{2} * C_{2} * C_{2}$. In this correspondence, hypermaps correspond to conjugacy classes of subgroups of $\Delta$, and hypermap coverings to subgroup inclusions. Towards the end of [9] the authors studied regular hypermaps with extra symmetries, namely, $G$-symmetric regular hypermaps for any subgroup $G$ of the outer automorphism $\operatorname{Out}(\Delta)$ of the triangle group $\Delta$. This can be viewed as an extension of the theory of regularity. In this paper we move in the opposite direction and restrict regularity to normal subgroups $\Theta$ of $\Delta$ of finite index. This generalises the notion of regularity to some non-regular objects.
\end{abstract}

\section{Introduction}

Regularity has always been present in geometry, often in the form of regular polyhedra or regular tessellations. Well known examples are the nine regular polyhedra comprising the five Platonic solids (the convex regular polyhedra) and the Kepler-Poinsot polyhedra. These polytopes can be viewed as regular maps, that is, cellular decompositions of closed surfaces. In the past two centuries there has been growing interest in highly symmetric maps mainly due to their connections with group theory, elliptic functions, the four colour problem, Riemann surfaces, Galois

Received February 3, 2005.

2000 Mathematics Subject Classification: 05C10, 05C25, 20B25, 20F65, 51E30, 57M07, 57M60.

Key words and phrases: hypermaps, maps, hypergraphs, regularity, restricted regularity, orientably regular.

Supported in part by UI\&D Matemática e aplicações of University of Aveiro, through Program POCTI of FCT co-financed by the European Community fund FEDER. 
theory and algebraic curves, among others. Excellent accounts linking regular maps with some different fields of mathematics can be found in the surveys of Jones [18], Jones and Singerman [21], Nedela [24] and Širán̆ [26]. After the famous Belyi theorem saying that "A Riemann surface $X$ is defined over the algebraic numbers $\overline{\mathbb{Q}}$ if and only if there is a covering (now called the Belyi function) from $X$ to $\overline{\mathbb{C}}$ unramified outside 0,1 and $\infty$ " [2] and the remarkable observation by Grothendieck [16] that the pre-image set $f^{-1}([0,1]) \subset X$ of a such Belyi function $f$ is a bipartite graph cellularly embedded on the surface $X$ (and hence a hypermap), there has been a growing interest in the hypermap theory.

Regularity has had different meanings in history. For example, uniformity (that is, vertices, edges and faces having constant valencies, say $l, m=2$ and $n$, respectively) has been taken for regularity in the early studies of regular polyhedra of genus one and two by Errera [14]. Although this weak form of regularity is enough to describe other "strong forms" of regularity in the sphere ("rotary", "reflexible"), this feature is exclusive to this surface; no other compact orientable surface has a similar behaviour. As noticed by Brahana in [3], for a surface of genus one (Anchor Ring) it gives only a certain measure of "regularity" (read "orientable regularity"). But Brahana himself took regularity to mean "orientable regularity" (that is, "rotary" or "orientable direct regularity"). There is a large number of papers on regular (or reflexible) maps, hypermaps and polytopes published in the 20th century. Most of the papers dealing with non-regularity are directly or indirectly dealing with some form of a lesser degree of regularity.

In this paper we introduce a restricted form of regularity that casts light upon some of the weaker forms of regularity studied earlier. In [22] and [9] one finds a study of regular maps and hypermaps with extra symmetries, namely, the $\operatorname{Out}(\Delta(\infty, \infty, 2))$-symmetric regular maps and $\operatorname{Out}(\Delta)$-symmetric regular hypermaps, respectively. These are regular (hyper)maps which are invariant with respect to the outer automorphisms. This can be viewed as an extension of the theory of regularity. In this paper we explore the opposite direction and propose to restrict the study of regularity to subgroups of $\Delta$. We set up the notion of $\Theta$ regularity for any normal subgroup $\Theta$ of $\Delta$ with finite index, widening this way the notion of regularity to include some (not all) non-regular hypermaps as "regular" hypermaps in some sense.

Reduced regularity is not at all new. An orientably regular map (or hypermap) is the most known restricted form of regularity, in this case restricted to orientation preserving automorphisms $\left(\Delta^{+}\right.$-regularity). 
Less familiar is the orientable bipartite-regularity that appeared in the medial maps of regular oriented maps studied by Archdeacon, Širán and Škoviera [1], an orientable regularity restricted to bipartite-facepreserving automorphisms. Among the 14 automorphism types of edgetransitive maps classified by Graver and Watkins [15], and referred by Siran, Tucker and Watkins in [27], 11 correspond to restrictly regular maps. Other forms of restricted regularity can be found in older literature, though not directly. Some maps appearing as geometrical illustrations of groups in Burnside's monograph [11] (and also in Dyck [13]) are non-regular (in the "reflexible" and "orientable" sense), and even nonuniform. For example, Fig. 10 in the Burnside's book [11] illustrates a non-regular map which is $\left\langle R_{2},\left(R_{0} R_{1}\right)^{3}\right\rangle^{\Delta}$-regular in our restricted sense of regularity.

The structure of the paper is organised as follows. In the last three subsections of the introductory part we give a brief introduction to the theory of hypermaps. For a deep introduction on maps/hypermaps we refer the reader to $[4,9,10,12,19,21]$. Section 2 is dedicated to $\Theta$-conservative hypermaps, a generalisation of "orientable hypermaps" $\left(\Delta^{+}\right.$-conservative hypermaps), and to $\Theta$-regularity; some classic results in the theory of hypermaps are generalised here. The main topic of section 3 is related to the monodromy group of a $\Theta$-conservative hypermap. Some concepts are generalised, for example, the "even word subgroup" $\operatorname{Mon}^{+}(\mathcal{H})$ of the monodromy group $\operatorname{Mon}(\mathcal{H})$ of an orientable hypermap $\mathcal{H}$ generalises to $\Theta$-word subgroup $\operatorname{Mon}^{\Theta}(\mathcal{H})$ of the monodromy group of a $\Theta$-conservative hypermap $\mathcal{H}$. The $\Theta$-monodromy group of a $\Theta$-conservative hypermap is introduced; relative to the even word subgroup $\operatorname{Mon}^{+}(\mathcal{H})=\langle R, L\rangle$, the $\Delta^{+}$-monodromy group is just the group generated by the restrictions of $R$ and $L$ to one of the two orbits inducing the two orientations of the underlying orientable surface. Section 4 deals with the notion of $\Theta$-marked hypermaps, a generalisation of "oriented hypermaps" $\left(\Delta^{+}\right.$-marked hypermaps). The relationship between $\Theta$-hypermaps and $\Theta$-conservative hypermaps is explored in section 5 . Section 6 introduces $\Theta$-slices, the $\Theta$-regions corresponding to "flags" $(\Delta$-slices $)$ in hypermaps and "darts" $\left(\Delta^{+}\right.$-slices $)$in oriented hypermaps. Section 7 illustrates, on an example, the construction of a regular $\Theta-$ marked hypermap with a given group as monodromy group. In section 8 we treat $\Theta$-regularity in terms of regular coverings and in section 9 we introduce $\Theta$-type and derive a formula for computing the characteristic of a $\Theta$-regular hypermap. The last section (section 10) is devoted to answering the question of when a hypermap can be "restrictly" regular 
or not. Families of non-regular maps that are restricly and not restrictly regular are given.

\subsection{Hypermaps}

A (algebraic) hypermap is a four-tuple $\mathcal{H}=\left(F ; r_{0}, r_{1}, r_{2}\right)$ consisting of a non-empty finite set $F$, the set of flags, and three permutations $r_{0}, r_{1}$, $r_{2}$ of $F$ satisfying $r_{0}^{2}=r_{1}^{2}=r_{2}^{2}=1$ and generating a permutation group $\operatorname{Mon}(\mathcal{H})=\left\langle r_{0}, r_{1}, r_{2}\right\rangle$, called the monodromy (or $\Delta$-monodromy) group of $\mathcal{H}$, that acts transitively on $F$. If one of the $r_{i}$ 's has fix points then $\mathcal{H}$ has boundary, otherwise $\mathcal{H}$ is boundary-free. The transitive action implies $|\operatorname{Mon}(\mathcal{H})| \geq|F|$. Every hypermap corresponds to a cellular embedding of a hypergraph $\mathcal{G}$ in $S$, also called a topological hypermap. Conversely, every topological hypermap can be described by a four-tuple $\mathcal{H}=\left(F ; r_{0}, r_{1}, r_{2}\right)$ introduced above.

A covering from $\mathcal{H}$ to $\mathcal{H}^{\prime}=\left(F^{\prime} ; s_{0}, s_{1}, s_{2}\right)$ is a function $\psi: F \rightarrow F^{\prime}$ satisfying $r_{i} \psi=\psi s_{i}$ for $i=0,1,2$. Due to the transitivity of the the actions, every covering is necessarily surjective. An isomorphism is an injective covering. If $\psi$ is a covering then the assignment $r_{i} \mapsto s_{i}$ extends to a canonical epimorphism from $\operatorname{Mon}(\mathcal{H})$ to $\operatorname{Mon}\left(\mathcal{H}^{\prime}\right)$. An automorphism of $\mathcal{H}$ is an isomorphism from $\mathcal{H}$ to $\mathcal{H}$, that is, a permutation of $F$ commuting with each $r_{i}$, hence commuting with every $g \in \operatorname{Mon}(\mathcal{H})$. The automorphisms of $\mathcal{H}$ form a group $\operatorname{Aut}(\mathcal{H})$ that acts semi regularly on $F$. As a consequence, $|\operatorname{Aut}(\mathcal{H})| \leq|F|$, and hence we have

$$
|\operatorname{Aut}(\mathcal{H})| \leq|F| \leq|\operatorname{Mon}(\mathcal{H})|
$$

An equality on one side implies an equality on the other side. This happens if and only if the action of $\operatorname{Aut}(\mathcal{H})$ on $F$ is regular. In this case $\mathcal{H}$ is called a regular hypermap.

The type of a hypermap $\mathcal{H}$ is a triple $(k ; l ; m)$, where $k, l$, and $m$ are the least common multiple of valencies of hypervertices, hyperedges and hyperfaces respectively. A map of type $\{m, k\}$ is a hypermap of type $(k ; 2 ; m)$. Denoting by $\mathcal{V}, \mathcal{E}$ and $\mathcal{F}$ the set of hypervertices, hyperedges and hyperfaces of a boundary-free hypermap $\mathcal{H}$ (respectively), then the characteristic $\chi(\mathcal{H})$ of $\mathcal{H}$, that is, the characteristic of the underlying surface, is given by the well known formula,

$$
\chi(\mathcal{H})=|\mathcal{V}|+|\mathcal{E}|+|\mathcal{F}|-\frac{|F|}{2} .
$$




\subsection{Finite permutation representation of hypermaps}

Let $\Delta$ denote the free product $C_{2} * C_{2} * C_{2}$ with presentation $\left\langle R_{0}\right.$, $R_{1}, R_{2}\left|R_{0}{ }^{2}=R_{1}{ }^{2}=R_{2}{ }^{2}=1\right\rangle$. There is a natural epimorphism $\rho: \Delta \longrightarrow$ $\operatorname{Mon}(\mathcal{H})$ taking $R_{i} \rightarrow r_{i}$. This epimorphism induces an action $w \cdot d:=$ $w d \rho$ of $\Delta$ on $F$. Fixing a flag $w \in F$, let $H$ be the stabiliser of $w$ in $\Delta$. Then $\Delta$ acts on the set of right cosets $\Delta / r H$ of $H$ in $\Delta$ by right multiplication, and $\rho$ induces a bijective function $\rho_{w}: \Delta / r H \longrightarrow F$, $H d \mapsto w \cdot d=w d \rho$. The kernel of $\rho$ is $H^{*}=H_{\Delta}$, the core of $H$ in $\Delta$. The group $\Delta / H^{*}$ acts transitively on $\Delta / r H$ by right multiplication ( $H d \cdot H^{*} g=H d H^{*} g=H d g$ ); this action is similar to the action of the monodromy group $\operatorname{Mon}(\mathcal{H})$ on $F$. Hence the hypermap $\mathcal{H}$ can be identified with $\left(\Delta /_{r} H ; H^{*} R_{0}, H^{*} R_{1}, H^{*} R_{2}\right)$, having monodromy group $\Delta / H_{\Delta} \cong \operatorname{Mon}(\mathcal{H})$. The subgroup $H$ of $\Delta$ is a fundamental subgroup of $\mathcal{H}$. This is independent up to a conjugation in $\Delta$. The automorphism group $\operatorname{Aut}(\mathcal{H})$ can be identified with $N_{\Delta}(H) / H$ acting on the set of right cosets $F=\Delta / r H$ (the set of flags) from the left, that is,

$$
\forall H g \in N_{\Delta}(H) / H, \forall d \in \Delta_{l} H, H d \cdot H g=H g^{-1} d .
$$

Any automorphism $\psi \in \operatorname{Aut}(\mathcal{H})$ is then a function $\phi_{g}: H d \mapsto H g^{-1} d$, for some $g \in N_{\Delta}(H)$. The semi-regularity of the action says that if $H d \phi_{g}=H d$ then $g \in H$.

The two groups $H^{*}=H_{\Delta}$, the core of $H$ in $\Delta$, and $H^{\Delta}=\langle H\rangle^{\Delta}$, the normal closure of $H$ in $\Delta$, give rise to two regular hypermaps, the covering core $\mathcal{H}_{\Delta}$ and the closure cover $\mathcal{H}^{\Delta}$, with the inclusions $H_{\Delta}<$ $H<H^{\Delta}$ determining a covering lattice $\mathcal{H}_{\Delta} \longrightarrow \mathcal{H} \longrightarrow \mathcal{H}^{\Delta}$.

\section{2. $\Theta$-conservative and $\Theta$-regular hypermaps}

Let $\Theta$ be a normal subgroup with finite index $n$ in $\Delta$. We say that a hypermap $\mathcal{H}$ is $\Theta$-conservative if its fundamental subgroup $H$ is a subgroup of $\Theta$. It is clear that if $\mathcal{H}$ is $\Theta$-conservative then so are its covering core $\mathcal{H}_{\Delta}$ and its closure cover $\mathcal{H}^{\Delta}$.

THEOREM 1. Let $\Theta$ be a normal subgroup of index $n$ in $\Delta$. If $\mathcal{H}$ is $\Theta$-conservative, then

(1) $\Theta$ acts by right multiplication on $F$ with $n$ orbits;

(2) the action of $\Theta$ on $F$ is uniform (all orbits have the same length).

Proof. (1) Naturally that $\Delta$ acts by right multiplication on $F=$ $\Delta / r H$ and, consequently, the subgroup $\Theta$ also acts by right multiplication 
on $F$. Each orbit (under the action of $\Theta$ ) is a set of the form $H d \Theta=$ $\{H d t \mid t \in \Theta\}$. Let $\mathcal{O}_{\Theta}$ denote the set of orbits $\{H d \Theta \mid H d \in F\}$. The function $\psi: \Delta / \Theta \longrightarrow \mathcal{O}_{\Theta}, \Theta d \mapsto H d \Theta$, is obviously onto, is well defined,

$$
\begin{aligned}
\Theta d_{1}=\Theta d_{2} & \Rightarrow d_{1}^{-1} d_{2}=\left(\left(d_{1} d_{2}^{-1}\right)^{-1}\right)^{d_{2}} \in \Theta, \quad \text { since } \Theta \triangleleft \Delta, \\
& \Rightarrow H d_{1}\left(d_{1}^{-1} d_{2}\right)=H d_{2} \in H d_{1} \Theta \\
& \Rightarrow H d_{1} \Theta=H d_{2} \Theta
\end{aligned}
$$

and is one-one,

$$
\begin{aligned}
H d_{1} \Theta=H d_{2} \Theta & \Rightarrow H d_{2}=H d_{1} g, \quad \text { for some } g \in \Theta, \\
& \Rightarrow \Theta d_{1} g d_{2}^{-1}=\Theta d_{1} d_{2}^{-1}=\Theta \\
& \Rightarrow \Theta d_{1}=\Theta d_{2} .
\end{aligned}
$$

(2) Let $H \Theta, H d_{1} \Theta, \ldots, H d_{n-1} \Theta$ be the $n$ orbits. As $H g d_{i}=H d_{i} d_{i}^{-1}$ $g d_{i}=H d_{i} g^{d_{i}} \in H d_{i} \Theta$, the function $\psi: H \Theta \longrightarrow H d_{i} \Theta, H g \mapsto H g d_{i}$ is clearly well defined and injective. For any $H d_{i} g \in H d_{i} \Theta, g^{d_{i}^{-1}} \in \Theta$ and so $H g^{d_{i}^{-1}} \psi=H g^{d_{i}^{-1}} d_{i}=H d_{i} g$, which shows that $\psi$ is onto.

These $n$ orbits, which can be understood as $n$ flag-colourings, will be called $\Theta$-orbits. If $\omega=H b$ is a flag, the $\Theta$-orbit determined by $\omega$ will be denoted indistinctly by $\omega \Theta$ or by $F_{b}^{\Theta}$. Denote by $F^{\Theta}$ the orbit $F_{1}^{\Theta}$ determined by the flag $H$. Notice that the normality of $\Theta$ in $\Delta$ allows us to write $F_{b}^{\Theta}=\{H t b \mid t \in \Theta\}$. The reason for the last notation is to bring it closer to the standard notation of $F^{+}$and $\mathrm{F}^{-}$ used in some literature to mean the two orbits $\omega \Delta^{+}$and $\omega r_{0} \Delta^{+}$of an orientable map/hypermap.

Some $\Theta$-conservative maps/hypermaps are very familiar although not under this name. For example, an orientable hypermap is a $\Delta^{+}$ conservative hypermap, where $\Delta^{+}$is the even word subgroup of $\Delta$, i.e., the normal closure $\left\langle R_{1} R_{2}, R_{2} R_{0}\right\rangle^{\Delta}$ in $\Delta$; a bipartite hypermap is a $\Delta^{\hat{0}}$-conservative hypermap, where $\Delta^{\hat{0}}$ is the normal closure $\left\langle R_{1}, R_{2}\right\rangle^{\Delta}$ of index 2 in $\Delta$; a pseudo-orientable hypermap (Wilson [28]) is a $\Delta^{0}$ conservative hypermap, where $\Delta^{0}$ is the normal closure $\left\langle R_{0}, R_{1} R_{2}\right\rangle^{\Delta}$, also a subgroup of index 2 in $\Delta$.

LEMMA 2. If $T=\left\{1=b_{1}, \ldots, b_{n}\right\}$ is a transversal for $\Theta$ in $\Delta$, then $F_{b_{1}}^{\Theta}, \ldots, F_{b_{n}}^{\Theta}$ are the $n$ orbits of the action of $\Theta$ on $F$.

Proof. The flags $H b_{1}, \ldots, H b_{n}$ belong to distinct orbits. In fact, for $t \in \Theta, H b_{i}=H b_{j} t$ implies that $b_{j} t b_{i}{ }^{-1} \in H \subset \Theta$, which implies that $\Theta b_{i}=\Theta b_{j} t \Leftrightarrow \Theta b_{i}=\Theta b_{j} \Leftrightarrow b_{i}=b_{j}$. 
The following theorem states that both the monodromy group and the automorphism group of a $\Theta$-conservative hypermap act on the set of $\Theta$-orbits $\mathcal{O}_{\Theta}$.

TheOREM 3. If $\mathcal{H}$ is a $\Theta$-conservative hypermap, then (1) the monodromy group $\operatorname{Mon}(\mathcal{H})$ acts transitively on the set of $\Theta$-orbits $\mathcal{O}_{\Theta}$ by right multiplication $\left(F_{b}^{\Theta} \cdot H_{\Delta} g=F_{b g}^{\Theta}\right)$, (2) the automorphism group $\operatorname{Aut}(\mathcal{H})$ acts (not necessarily transitively) on $\mathcal{O}_{\Theta}$ by $\omega \Theta \psi=\omega \psi \Theta$.

Proof. (1) For any $g \equiv H_{\Delta} g \in \Delta / r H_{\Delta}=\operatorname{Mon}(\mathcal{H})$ and for any orbit $F_{b}^{\Theta} \in \mathcal{O}_{\Theta}$,

$$
\begin{aligned}
F_{b}^{\Theta} \cdot g=F_{b}^{\Theta} H_{\Delta} g & =\{H b t g \mid t \in \Theta\} \\
& =\left\{H b g t^{\prime} \mid t^{\prime}=t^{g} \in \Theta^{g}=\Theta\right\} \\
& =F_{b g}^{\Theta} .
\end{aligned}
$$

(2) Let $\omega=H b \in \Delta_{r} H$ be a flag and $\omega \cdot \Theta=F_{b}^{\Theta}$ be the $\Theta$-orbit determined by $\omega$. For any automorphism $\psi \in \operatorname{Aut}(\mathcal{H})=N_{\Delta}(H) / H, \psi$ is a function $\phi_{g}: H b \mapsto H g^{-1} b$, for some $g \in N_{\Delta}(H)$. Since $H b \Theta \phi_{g}=$ $H g^{-1} b \Theta$ it follows $F_{b}^{\Theta} \phi_{g}=F_{g^{-1} b}^{\Theta}$, that is, $\omega \Theta \psi=\omega \psi \Theta$.

Denote by $\operatorname{Aut}^{\Theta}(\mathcal{H})$ the subset of $\operatorname{Aut}(\mathcal{H})$ consisting of the automorphisms preserving each $\Theta$-orbit $F_{b_{i}}^{\Theta}, i=1, \ldots, n$. This group will be called the $\Theta$-automorphism group of $\mathcal{H}$.

Theorem 4. $A u t^{\Theta}(\mathcal{H})=N_{\Theta}(H) / H$.

Proof. If $\phi=H \tau \in N_{\Theta}(H) / H(\tau \in \Theta)$, then $\phi$ preserves each $\Theta$ orbit $F_{b}^{\Theta}$; in fact, $F_{b}^{\Theta} \phi=F_{\tau^{-1} b}^{\Theta}=\left\{H t^{\prime} b \mid t^{\prime}=t \tau^{-1} \in \Theta\right\}=F_{b}^{\Theta}$. If $\phi=H g \in \operatorname{Aut}^{\Theta}(\mathcal{H}) \subset \operatorname{Aut}(\mathcal{H})=N_{\Delta}(H) / H$, then $\phi$ preserves each orbit $F_{b}^{\Theta} \in \mathcal{O}_{\Theta}$. In particular $\phi$ preserves the orbit $F_{1}^{\Theta}$ determined by $H$, and so, $H \phi \in F_{1}^{\Theta}$, that is, $H g^{-1}=H t$ for some $t \in \Theta$. Hence $g \in \Theta$ and $\phi=H g \in N_{\Theta}(H) / H$.

We say that $\mathcal{H}$ is $\Theta$-regular if $\operatorname{Aut}^{\Theta}(\mathcal{H})$ acts transitively on each $\Theta$ orbit $F_{b_{1}}^{\ominus}, \ldots, F_{b_{n}}^{\ominus}$.

Theorem 5. If $A u t^{\Theta}(\mathcal{H})$ acts transitively on a $\Theta$-orbit $F_{b_{i}}^{\Theta}$, then $A u t^{\Theta}(\mathcal{H})$ acts transitively on any other $\Theta$-orbit.

Proof. Let us show that if $\operatorname{Aut}^{\Theta}(\mathcal{H})$ acts transitively on the orbit $F_{d}^{\ominus}$ then $\operatorname{Aut}^{\Theta}(\mathcal{H})$ acts transitively on the orbit $F_{1}^{\Theta}$. For any $t \in \Theta$, there 
is $\phi_{g}=H g \in N_{\Theta}(H) / H=\operatorname{Aut}^{\Theta}(\mathcal{H})$ such that $H d\left(d^{-1} t d\right)=H d \phi_{g}=$ $H g^{-1} d$, that is, $H t=H g^{-1}=H \phi_{g}$. Hence $\operatorname{Aut}^{\Theta}(\mathcal{H})$ acts transitively on $F_{1}^{\Theta}$. To finish the proof we show that if $\operatorname{Aut}^{\Theta}(\mathcal{H})=N_{\Theta}(H) / H$ acts transitively on the orbit $F_{1}$ then it acts transitively on any orbit $F_{d}^{\Theta}$. For any $t \in \Theta$, there is $\phi_{g}=H g \in N_{\Theta}(H) / H=\operatorname{Aut}^{\Theta}(\mathcal{H})$ such that $H\left(d t d^{-1}\right)=H \phi_{g}=H g^{-1}$. But this is equivalent to $H d t=H g^{-1} d=$ $H d \phi_{g}$.

THEOREM 6. $\mathcal{H}$ is $\Theta$-regular if and only if $H$ is a normal subgroup of $\Theta$.

Proof. $(\Leftrightarrow)$ If $H \triangleleft \Theta$, then $N_{\Theta}(H)=\Theta$ and so, for any $t \in \Theta, H t=$ $H \phi_{t^{-1}}$, that is, $\Theta / H=N_{\Theta}(H) / H=\operatorname{Aut}^{\Theta}(\mathcal{H})$ acts transitively on the orbit $F_{1}^{\Theta}$.

$\Leftrightarrow$ If $\operatorname{Aut}^{\Theta}(\mathcal{H})=N_{\Theta}(H) / H$ acts transitively on the orbit $F_{1}^{\Theta}$, then for any $t \in \Theta$, there is $g \in N_{\Theta}(H)$ such that $H t=H \phi_{g}=H g^{-1}$. This says that $t \in H g^{-1} \subset H N_{\Theta}(H)=N_{\Theta}(H)$. Hence $\Theta \subset N_{\Theta}(H) \Leftrightarrow$ $N_{\Theta}(H)=\Theta$.

Often, regularity in map/hypermap theory appears in the form of $\Delta$-regular (or simply regular) and $\Delta^{+}$-regular. Depending on the context, $\Delta^{+}$-regular has been known as "rotary", "directly regular" or "orientably regular". Less usual is $\Delta^{0}$-regular, or "bipartite-regular".

\section{3. $\Theta$-monodromy group}

The action of $\Theta$ on the set of flags $F$ of a $\Theta$-conservative hypermap $\mathcal{H}$ induces a natural homomorphism (not onto) $\alpha$ from $\Theta$ to the symmetric group $\operatorname{Sym}(F)$. The image $\operatorname{Im}(\alpha)=\Theta \alpha$ is a subgroup of $\operatorname{Mon}(\mathcal{H})$. Call it the $\Theta$-word subgroup and denote it by $\operatorname{Mon}^{\ominus}(\mathcal{H})$. In an orientable hypermap the $\operatorname{Mon}^{\Delta^{+}}(\mathcal{H})$ is just the "even" word subgroup generated by the "even" words $H_{\Delta} R_{1} R_{2}, H_{\Delta} R_{2} R_{0}$.

LEMma 7. $\operatorname{Mon}^{\ominus}(\mathcal{H}) \cong \Theta / H_{\Delta}$ and $\operatorname{Mon}^{\ominus}(\mathcal{H}) \triangleleft_{n} \operatorname{Mon}(\mathcal{H})$.

Proof. The kernel $\operatorname{Ker}(\alpha)$ is the core of $H$ in $\Delta$. Moreover, $\Theta / H_{\Delta} \triangleleft_{n}$ $\Delta / H_{\Delta} \cong \operatorname{Mon}(\mathcal{H})$.

Now, $\Theta$ also acts (by right multiplication) on each $\Theta$-orbit $F_{b}^{\ominus}(b \in$ $T$ ). This gives rise to a homomorphism $\alpha_{b}: \Theta \longrightarrow \operatorname{Sym}\left(F_{b}^{\Theta}\right), t \longmapsto$ $t \alpha_{b}: H b x \mapsto H b x t$. We denote by $\Theta-\operatorname{Mon}_{b}(\mathcal{H})$ the subgroup $\operatorname{Im}\left(\alpha_{b}\right)=$ 
$\Theta \alpha_{b}<\operatorname{Sym}\left(F_{b}^{\Theta}\right)$ and call it the $\Theta$-monodromy group of $\mathcal{H}$ on $F_{b}^{\Theta}$. In particular, the $\Theta$-monodromy of $\mathcal{H}$ on the orbit $F_{1}^{\Theta}$ will be simply called $\Theta$-monodromy group of $\mathcal{H}$ and will be denoted by $\Theta-\operatorname{Mon}(\mathcal{H})$.

THEOREM 8. $\Theta-\operatorname{Mon}_{b}(\mathcal{H})$ is isomorphic to $\Theta / H_{\Theta}^{b}$.

Proof. In fact, the kernel $\operatorname{Ker}\left(\alpha_{b}\right)$ is the core of $H^{b}$ in $\Theta$.

On different $\Theta$-orbits the action of $\Theta$ may not be equivalent, however, they induce isomorphic groups.

THEOREM 9. For any $b \in T, \quad \Theta-M_{b}(\mathcal{H})$ is isomorphic to $\Theta$ $\operatorname{Mon}(\mathcal{H}) \cong \Theta / H_{\Theta}$.

Proof. In fact, from the normality of $\Theta$ in $\Delta$ we deduce

$$
\Theta-\operatorname{Mon}_{b}(\mathcal{H}) \cong \Theta / H_{\Theta}^{b}=\left(\Theta / H_{\Theta}\right)^{b} \cong \Theta / H_{\Theta} \cong \Theta-\operatorname{Mon}(\mathcal{H}) \text {. }
$$

Corollary 10. If $\mathcal{H}$ is $\Theta$-regular, then (1) $\Theta$-Mon $(\mathcal{H}) \cong \Theta / H$; (2) $|F|=|\Delta: \Theta||\Theta-M o n(\mathcal{H})|=n|\Theta-M o n(\mathcal{H})|$.

The semi-regular action of $\operatorname{Aut}^{\Theta}(\mathcal{H})$ and the transitive action of $\Theta$-Mon $(\mathcal{H})$ on the set of $\Theta$-orbits $F^{\Theta}$ of a $\Theta$-conservative hypermap $\mathcal{H}$ give the following chain of inequalities,

$$
\left|\operatorname{Aut}^{\Theta}(\mathcal{H})\right| \leq\left|F^{\ominus}\right| \leq|\Theta-\operatorname{Mon}(\mathcal{H})| .
$$

THEOREM 11. The following statements are equivalent:

(1) $\mathcal{H}$ is $\Theta$-regular; (2) $\left|A u t^{\Theta}(\mathcal{H})\right|=\left|F^{\Theta}\right|$; (3) $\left|F^{\Theta}\right|=|\Theta-M o n(\mathcal{H})|$.

Proof. $\mathcal{H}$ is $\Theta$-regular $\Leftrightarrow H \triangleleft \Theta \Leftrightarrow H_{\Theta}=H \Leftrightarrow N_{\Theta}(H) / H=\Theta / H$. By Theorem 9, $\Theta-\operatorname{Mon}(\mathcal{H}) \cong \Theta / H_{\Theta}$ and by Theorem $4, \operatorname{Aut}^{\Theta}(\mathcal{H})=$ $N_{\Theta}(H) / H$. Hence $\mathcal{H}$ is $\Theta$-regular $\Leftrightarrow \operatorname{Aut}^{\Theta}(\mathcal{H}) \cong \Theta / H \Leftrightarrow \mid$ Aut $^{\Theta}(\mathcal{H}) \mid=$ $\left|F^{\Theta}\right|$. Similarly, $\left|F^{\Theta}\right|=|\Theta-\operatorname{Mon}(\mathcal{H})| \Leftrightarrow|\Theta: H|=\left|\Theta: H_{\Theta}\right| \Leftrightarrow H_{\Theta}=H$ $\Leftrightarrow \mathcal{H}$ is $\Theta$-regular.

For each $t \in \Theta$, the permutation $t \alpha_{b}$ is essentially the action of $t$ on $F_{b}^{\Theta}$. Hence it makes sense to denote by $t_{\left.\right|_{F_{b}^{\Theta}}}$ the permutation $t \alpha_{b}$. Having this in mind, we can write

$$
\Theta-\operatorname{Mon}(\mathcal{H})=\Theta_{\left.\right|_{F} \Theta}
$$

and rewrite theorem 9 as follows: for each orbit $F_{b}^{\Theta}, \quad \Theta_{\left.\right|_{F_{b}^{\Theta}}} \cong \Theta_{\left.\right|_{F} \Theta}$. 


\section{4. $\Theta$-marked hypermaps}

As $\Theta$ has finite index in a finite generated group $\Delta$, by NielsenSchreier theorem $\Theta$ is also finitely generated. Moreover, as $\Delta$ is a free product $C_{2} * C_{2} * C_{2}$, by Kurosh theorem (Proposition 3.6, p.120 of [23]) $\Theta$ is also a free product $\Theta \cong C_{2} * \cdots * C_{2} * C_{\infty} * \cdots * C_{\infty}$, for a certain number of factors $C_{2}$ and $C_{\infty}$, where the number of factors of one type $\left(C_{2}\right.$ or $\left.C_{\infty}\right)$ may be empty. According to the above free-product decomposition (which is unique up to a permutation of its factors),

$$
\Theta=\left\langle a_{1}, \ldots, a_{s}, z_{1}, \ldots, z_{t} \mid a_{i}^{2}=1, i=1 \ldots s\right\rangle .
$$

Let $\operatorname{rank}(\Theta)=m$. For simplicity, put $\left\{x_{1}, \ldots, x_{m}\right\}=\left\{a_{1}, \ldots, a_{s}, z_{1}\right.$, $\left.\ldots, z_{t}\right\}$. If there are factors $C_{2}$ in the above free-product decomposition, we write them first, so we expect involutions coming first in the above generator's set.

By a $\Theta$-marked hypermap we mean a $(m+1)$-tuple

$$
\mathcal{Q}=\left(\Omega ; \alpha_{1}, \ldots, \alpha_{m}\right),
$$

where $\Omega$ is a finite set, $\alpha_{1}, \ldots, \alpha_{m}$ are permutations of $\Omega$ generating a group $G$ acting transitively on $\Omega$ such that the function $\rho: x_{i} \mapsto \alpha_{i}$ extends to an epimorphism from $\Theta$ to $G$. This group $G$ is the monodromy group of $\mathcal{Q}$ and will be denoted by $\operatorname{Mon}(\mathcal{Q})$. The name "marked hypermap" was chosen because the triple $(G, \Omega, D)$, where $D=\left\{\alpha_{1}, \ldots, \alpha_{m}\right\}$, is a marked finite transitive permutation group (Singerman [25]). The epimorphism $\rho$ induces a transitive action of $\Theta$ on $\Omega$ defined by $w \cdot d:=$ $w \cdot(d \rho)$, for all $d \in \Theta$. For a fixed $w \in \Omega$, let $Q$ be the stabiliser of $w$ in $\Theta$. Then $\Theta$ acts by right multiplication on the right cosets $\Theta / r Q$ and $\rho$ induces a bijective function $\rho_{w}: \Theta / r Q \longrightarrow \Omega, Q d \mapsto w d \rho$. The kernel of $\rho$ is the core $Q_{\Theta}$ of $Q$ in $\Theta$. The group $\Theta / Q_{\Theta}$ (which is isomorphic to G by $\rho$ ) acts transitively on $\Theta / r Q$ by right multiplication $\left(Q d \cdot Q_{\Theta} g=Q d g\right)$ and this action is similar to the action of $G=\operatorname{Mon}(\mathcal{Q})$ on $\Omega$; that is, the following diagram

$$
\begin{array}{cc}
\Theta / r Q \times \Theta / Q_{\Theta} & \longrightarrow \Theta / r Q \\
\rho_{w} \downarrow \rho & \\
\Omega \times G & \downarrow \rho_{w} \\
\Omega & \Omega
\end{array}
$$

commutes. The $\Theta$-marked hypermap $\mathcal{Q}$ can then be identified with the $\Theta$-marked hypermap $\left(\Theta / r Q ; Q_{\Theta} x_{1}, \ldots, Q_{\Theta} x_{m}\right)$ with monodromy group $\Theta / Q_{\Theta} \cong \operatorname{Mon}(\mathcal{Q})$. This subgroup $Q$ of $\Theta$ will be a called a $\Theta$-marked fundamental subgroup of $\mathcal{Q}$. Giving two $\Theta$-marked hypermaps $\mathcal{Q}_{1}=$ 
$\left(\Omega_{1} ; \alpha_{1}, \ldots, \alpha_{m}\right)$ and $\mathcal{Q}_{2}=\left(\Omega_{2} ; \beta_{1}, \ldots, \beta_{m}\right)$, a covering from $\mathcal{Q}_{1}$ to $\mathcal{Q}_{2}$ is a function $\phi: \Omega_{1} \longrightarrow \Omega_{2}$ (necessarily onto by the transitive action) such that for any $w \in \Omega_{1}, w \alpha_{i} \phi=w \phi \beta_{i}$, for $i=1, \ldots, m$. An isomorphism $\phi: \mathcal{Q}_{1} \longrightarrow \mathcal{Q}_{2}$ is just a one-to-one covering and an automorphism of a $\Theta$-marked hypermap $\mathcal{Q}=\left(\Omega ; \alpha_{1}, \ldots, \alpha_{m}\right)$ is an isomorphism from $\mathcal{Q}$ to $\mathcal{Q}$, that is, a permutation of $\Omega$ commuting with each $\alpha_{i}(i=1, \ldots, m)$. The above observations prove:

THEOREM 12. If $\mathcal{Q}=\left(\Omega ; \alpha_{1}, \ldots, \alpha_{m}\right)$ is a $\Theta$-marked hypermap, then

$$
\mathcal{Q} \cong\left(\Theta / r Q ; Q_{\ominus} x_{1}, \ldots, Q_{\Theta} x_{m}\right),
$$

where $Q$ is the stabiliser in $\Theta$ of any $w \in \Omega$.

As we can observe from the above theorem, the $\Theta$-marked fundamental subgroup $Q$ of $\mathcal{Q}$ is independent (up to an isomorphism) from the fixed $w$; different choices of $w$ give rise to conjugate $\Theta$-marked fundamental subgroups (conjugation in $\Theta$ ), giving rise to isomorphic $\Theta$ marked hypermaps. In fact, giving any conjugate $Q^{t}(t \in \Theta)$ the function $\Theta / r Q \rightarrow \Theta / r Q^{t}$ defined by $Q d \mapsto t^{-1} Q d=Q^{t} t^{-1} d$ is a bijection commuting with each $Q_{\Theta} x_{i}$. The group of automorphisms $\operatorname{Aut}(\mathcal{Q})$ of $\mathcal{Q}$ is isomorphic to the quotient group $N_{G}\left(\operatorname{Stab}_{G}(w)\right) / \operatorname{Stab}_{G}(w)$ (Singerman [25]). If $Q$ is the stabiliser of $w$ in $\Theta$, then $\operatorname{Aut}(\mathcal{Q}) \cong N_{\Theta}(Q) / Q$ (Zassenhaus [29], p.51). Definitions and results proved specifically for $\Delta$-marked hypermaps (that is, hypermaps) and $\Delta^{+}$-marked hypermaps (that is, oriented hypermaps, see for instance $[6,7,8]$ ) can be adapted to $\Theta$-marked hypermaps. For example, the group of automorphisms Aut $(\mathcal{Q})$ acts semi-regularly on $\Omega$ giving the double inequality

$$
|\operatorname{Aut}(\mathcal{Q})| \leq|\Omega| \leq|\operatorname{Mon}(\mathcal{Q})|
$$

If $\operatorname{Aut}(\mathcal{Q})$ acts transitively (hence regularly) on $\Omega$, then we say that $\mathcal{Q}$ is regular. As $\operatorname{Aut}(\mathcal{Q}) \cong N_{\Theta}(Q) / Q$ and $\Omega$ is equipotent to $\Theta / r Q$, $\mathcal{Q}$ is regular if and only if $Q \triangleleft \Theta$. Moreover, an equality on one side of the above double inequalities implies an equality on the other side. Consequently, the following are equivalent: (1) $\mathcal{Q}$ is regular (2) $Q \triangleleft \Theta$, (3) $|\operatorname{Aut}(\mathcal{Q})|=|\Omega|,(4)|\operatorname{Mon}(\mathcal{Q})|=|\Omega|$.

\section{5. $\Theta$-marked hypermaps versus $\Theta$-conservative hypermaps}

Any $\Theta$-conservative hypermap $\mathcal{H}=\left(F ; r_{0}, r_{1}, r_{2}\right)$, with fundamental subgroup $H<\Theta$, gives rise to $n \Theta$-marked hypermaps

$$
\mathcal{H}_{b}^{\Theta}=\left(F_{b}^{\Theta} ; x_{\left.1\right|_{F_{b}}}, \ldots, x_{\left.m\right|_{F_{b}} ^{\Theta}}\right),
$$


one for each $\Theta$-orbit $F_{b}^{\Theta}$. These special $\Theta$-marked hypermaps will be called $\Theta$-hypermaps. Each $\Theta$-hypermap $\mathcal{H}_{b}^{\Theta}$ will be called a "b-image" of

$$
\mathcal{H}^{\Theta}=\left(F^{\Theta} ; x_{\left.1\right|_{F} \Theta}, \ldots, x_{\left.m\right|_{F} ^{\Theta}}\right) \text {. }
$$

The monodromy group of $\mathcal{H}_{b}^{\Theta}$ is the $\Theta$-monodromy group of $\mathcal{H}$ on $F_{b}^{\Theta}$,

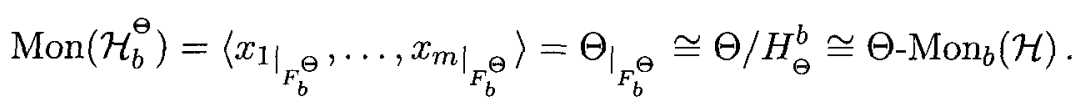

As $\Theta / H_{\Theta}^{b} \cong \Theta / H_{\Theta} \cong \operatorname{Mon}\left(\mathcal{H}^{\Theta}\right)=\left\langle x_{\left.1\right|_{F} \Theta}, \ldots, x_{\left.m\right|_{F} \Theta}\right\rangle$, the $\Theta$-hypermap $\mathcal{H}^{\Theta}$ and its $b$-images $\mathcal{H}_{b}^{\Theta}, b \in T$, all have the same monodromy group,

$$
\operatorname{Mon}\left(\mathcal{H}_{b}^{\Theta}\right) \cong \operatorname{Mon}\left(\mathcal{H}^{\Theta}\right) \text {. }
$$

However $\mathcal{H}^{\Theta}$ may not be isomorphic to any of its $b$-images $\mathcal{H}_{b}^{\ominus}$. If $\mathcal{H}$ is $\Theta$-regular this translates to "the two monodromy groups $\operatorname{Mon}\left(\mathcal{H}_{b}^{\Theta}\right)$ and $\operatorname{Mon}\left(\mathcal{H}^{\Theta}\right)$ may not be monodromically isomorphic in respect to their fixed set of generators; that is, the function $x_{\left.i\right|_{F} \Theta} \mapsto x_{\left.i\right|_{F_{b}}}, i=1, \ldots, m$, may not extend to an isomorphism". In the next theorem we shall show that if $\mathcal{H}$ is $\Theta$-regular then $\mathcal{H}^{\Theta}$. is isomorphic to all its $b$-images if and only if $\mathcal{H}$ is $\Delta$-regular.

Theorem 13. A $\Theta$-regular hypermap $\mathcal{H}$ is $\Delta$-regular if and only if for any $b \in T$, the function $\Theta_{\left.\right|_{F} \Theta} \longrightarrow \Theta_{\left.\right|_{F_{b}^{\Theta}}}, x_{\left.\right|_{F} \Theta} \mapsto x_{\left.\right|_{F_{b}^{\Theta}}}$, is an isomorphism.

Proof. $(\Rightarrow)$ Let $\mathcal{H}$ be $\Delta$-regular, which is the same as $H \triangleleft \Delta$. Then the function

is well defined and injective; in fact,

$$
\begin{aligned}
\phi: \Theta_{\left.\right|_{F} \Theta} & \longrightarrow \Theta_{\left.\right|_{F_{b}^{\Theta}}} \\
x_{\left.\right|_{F} \Theta} & \longrightarrow x_{\left.\right|_{F_{b}}}
\end{aligned}
$$

$$
\begin{aligned}
x_{\left.1\right|_{F} ^{\Theta}}=x_{\left.2\right|_{F} \Theta} & \Leftrightarrow \forall t \in \Theta, H t x_{1}=H t x_{2} \\
& \Leftrightarrow \forall t \in \Theta, x_{1} x_{2}{ }^{-1} \in H^{t}=H^{b t}(=H), \text { since } H \triangleleft \Delta, \\
& \Leftrightarrow \forall t \in \Theta, H b t x_{1}=H b t x_{2} \\
& \Leftrightarrow x_{\left.1\right|_{F_{b}}}=x_{\left.2\right|_{F_{b}}} .
\end{aligned}
$$

$\phi$ is obviously onto. Since $x_{\left.\right|_{A}} y_{\left.\right|_{A}}=(x y)_{\left.\right|_{A}}$ the function $\phi$ is obviously a homomorphism. 
$(\Leftarrow)$ If, for any $b \in T$, the function

$$
\begin{aligned}
\phi: \Theta_{F_{F}^{\Theta}} & \longrightarrow \Theta_{\left.\right|_{F_{b}^{\Theta}}} \\
x_{\left.\right|_{F} \Theta} & \longrightarrow x_{\left.\right|_{F_{b}^{\Theta}}}
\end{aligned}
$$

is an isomorphism then, in particular, it is well defined. As for each $h \in$ $H$, the permutation $h_{\left.\right|_{F}}: F^{\Theta} \longrightarrow F^{\Theta}, H t \mapsto H t h=t H h=t H=H t$, is the identity permutation, then for any $b \in T$,

$$
\begin{aligned}
1_{\left.\right|_{F_{b}^{\Theta}}}=h_{\left.\right|_{F_{b}^{\Theta}}} & \Leftrightarrow \forall t \in \Theta, H b t=H b t h \\
& \Leftrightarrow \forall t \in \Theta, h \in H^{b t} \\
& \Rightarrow h \in H^{b}, \quad \text { by taking } t=1 .
\end{aligned}
$$

Therefore, $H \subset H^{b}$ for any $b \in T$. As for any $d \in \Delta, d=t b$ for some $b \in T$ and $t \in \Theta$, then $H^{d}=H^{t b}=H^{b}$ and so $H \subset H^{d}$ for any $d \in \Delta$. Hence $H \triangleleft \Delta$.

COROLlary 14. A $\Theta$-regular hypermap $\mathcal{H}$ is $\Delta$-regular if and only if for any orbit $F_{b}^{\Theta}$, the function $\Theta_{\left.\right|_{F} ^{\Theta}} \longrightarrow \Theta_{\left.\right|_{F_{b}^{\Theta}}}, x_{\left.i\right|_{F} \Theta} \mapsto x_{\left.i\right|_{F_{b}^{\Theta}}}$, for $i=1, \ldots, m$, extends to an isomorphism.

Corollary 15. Let $T=\left\{1=b_{1}, \ldots, b_{n}\right\}$ be a transversal for $\Theta$ in $\Delta$. A $\Theta$-regular hypermap $\mathcal{H}$ is $\Delta$-regular if and only if the $\Theta$-hypermaps $\mathcal{H}_{b_{1}}^{\ominus}, \ldots, \mathcal{H}_{b_{n}}^{\Theta}$ are all isomorphic (that is, if and only if $\mathcal{H}$ is isomorphic to all its b-images).

More generally, replacing $\Delta$ by a normal subgroup $\Pi$ of $\Delta$ we get the following more general result:

Corollary 16. If $\Theta<\Pi<\Delta$ (with both $\Theta \triangleleft \Delta$ and $\Pi \triangleleft \Delta$ ) and $T^{\Pi}=\left\{1=b_{1}, \ldots, b_{n}\right\}$ is a transversal for $\Theta$ in $\Pi$, then a $\Theta$-regular hypermap is $\Pi$-regular if and only if the $\Theta$-hypermaps $\mathcal{H}_{b_{1}}^{\Theta}, \ldots, \mathcal{H}_{b_{n}}^{\Theta}$ are all isomorphic.

Although the $\Theta$-orbits of a $\Theta$-conservative hypermap $\mathcal{H}$ all have the same length, the action of $\Theta$ on them may not all be equivalent. Even if $\mathcal{H}$ is $\Theta$-regular this may not be sufficient for $\Theta$ acting equivalently on all $\Theta$-orbits. Let $\mathcal{H}$ be $\Theta$-regular. For each $b \in T$, the bijective function 
$\varphi_{b}: F^{\Theta} \longrightarrow F_{b}^{\Theta}, H t \mapsto H t b$, induces a commutative diagram

$$
\begin{array}{cc}
F^{\Theta} \times \Theta & F^{\Theta} \\
\varphi_{b} \downarrow_{i d} & \downarrow^{\varphi_{b}} \\
F_{b}^{\Theta} \times \Theta & \longrightarrow F_{b}^{\Theta}
\end{array}
$$

if and only if $\mathcal{H}^{\Theta}$ is isomorphic to its $b$-image $\mathcal{H}_{b}^{\Theta}$. By Corollary $15, \Theta$ acts equivalently on all orbits if and only if $\mathcal{H}$ is $\Delta$-regular. This proves:

Theorem 17. Let $\mathcal{H}$ be a $\Theta$-regular hypermap. The action of $\Theta$ on the $\Theta$-orbits are all equivalent if and only if $\mathcal{H}$ is $\Delta$-regular.

Let $b$ be an element of a transversal $T$ for $\Theta$ in $\Delta$. Being $\Theta$ normal in $\Delta$, the $\Theta$-orbit $F_{b}^{\Theta}=\{H b t \mid t \in \Theta\}$ can be written as the set $\Theta / r H \cdot b=$ $\{H t b \mid t \in \Theta\}$. The conjugate $H^{b}$ is a subgroup of $\Theta$ and the set of right cosets $\Theta / r H^{b}=\left(\Theta_{h} H\right)^{b}$ is equipotent to the set of right cosets $\Theta_{/ r} H$, which is equipotent to $\Theta / r H \cdot b=F_{b}^{\Theta}$. Hence the function

$$
H b t \mapsto H^{b} t
$$

is a bijection from $F_{b}^{\Theta}$ to $\Theta / H^{b}$. Moreover, the right action $H b t$. $\tau_{F_{b}^{\Theta}}:=H b t \tau$ of $\operatorname{Mon}\left(\mathcal{H}_{b}^{\Theta}\right)$ on $F_{b}^{\Theta}$ is equivalent to the right action $H^{b} t \cdot H_{\Theta}^{b} \tau=H^{b} t \tau$ of $\Theta / H_{\Theta}^{b}$ on $\Theta / r H^{b}$. Hence the b-image $\mathcal{H}_{b}^{\Theta}=$ $\left(F_{b}^{\Theta} ; x_{\left.1\right|_{F_{b}} ^{\Theta}}, \ldots, x_{\left.m\right|_{F_{b}^{\Theta}}}\right)$ is isomorphic to the $\Theta$-marked hypermap

$$
\mathcal{Q}\left(H^{b}\right)=\left(\Theta / r H^{b} ; H_{\Theta}^{b} x_{1}, \ldots, H_{\Theta}^{b} x_{m}\right) .
$$

with $\Theta$-marked fundamental subgroup $H^{b}$.

Reciprocally, if $\mathcal{Q}=\left(\Omega ; \alpha_{1}, \ldots, \alpha_{m}\right)$ is a $\Theta$-marked hypermap, as seen earlier $\mathcal{Q}$ is isomorphic to $\left(\Theta /{ }_{r} Q Q_{\Theta} x_{1}, \ldots, Q_{\Theta} x_{m}\right)$ for some $\Theta$-marked fundamental subgroup $Q<\Theta$. Let $\mathcal{H}$ be the hypermap $\left(\Delta /{ }^{\prime} Q ; Q_{\Delta} R_{0}, Q_{\Delta} R_{1}, Q_{\Delta} R_{2}\right)$, called here the $\Delta$-form of $\mathcal{Q}$. Then $\mathcal{H}$ is $\Theta$-conservative and

$$
\mathcal{H}^{\Theta} \cong\left(\Theta / r Q ; Q_{\Theta} x_{1}, \ldots, Q_{\Theta} x_{m}\right) \cong \mathcal{Q} .
$$

LEMma 18. Let $Q$ and $K$ be subgroups of $\Theta$ and let $g \in \Theta$. Then $\gamma_{g}: \Theta / r Q \longrightarrow \Theta_{r} K, Q t \mapsto K g t$, is well defined if and only if $Q \subset K^{g}$.

TheOREM 19. Let $\mathcal{Q}=\left(\Theta / r Q ; Q_{\Theta} x_{1}, \ldots, Q_{\Theta} x_{m}\right)$ and $\mathcal{K}=(\Theta / r K$; $\left.K_{\Theta} x_{1}, \ldots, K_{\Theta} x_{m}\right)$ be two $\Theta$-marked hypermaps. Then $\mathcal{Q}$ covers $\mathcal{K}$ if and only if $Q<K^{g}$ for some $g \in \Theta$. 
Proof. ( $\Rightarrow$ ): If $\psi: \Theta / r Q \longrightarrow \Theta / r K$ is a function commuting the respective actions, then $Q \psi=K g$, for some $g \in \Theta$. Then, for all $t \in \Theta$, $Q t \psi=Q Q_{\Theta} t \psi=Q \psi K_{\Theta} t=K g t$, that is, $\psi=\gamma_{g}$. By Lemma 18, $Q<K^{g}$.

$(\Leftarrow)$ : Reciprocally, if $Q<K^{g}$, for some $g \in \Theta$, then $\gamma_{g}$, as defined by Lemma 18 is well defined and determines a covering $\mathcal{Q} \rightarrow \mathcal{K}$. In fact, $Q<K^{g}$ implies that $Q_{\Theta}<K_{\Theta}$, then for all $t \in \Theta$ and $x \in$ $\left\{x_{1}, \ldots, x_{n}\right\}, Q t Q_{\Theta} x \gamma_{g}=Q t x \gamma_{g}=K g t x=K g t K_{\Theta} x=Q t \gamma_{g} K_{\Theta} x$. Hence $\gamma_{g}$ commutes with both actions .

Let $\mathcal{Q}=\left(\Theta / r Q ; Q_{\Theta} x_{1}, \ldots, Q_{\Theta} x_{m}\right)$ be a $\Theta$-marked hypermap with $\Theta$ marked fundamental subgroup $Q$. As seen above, $\mathcal{Q} \cong \mathcal{H}^{\Theta}$, where $\mathcal{H}=$ $\left(\Delta / r Q ; Q_{\Delta} R_{0}, Q_{\Delta} R_{1}, Q_{\Delta} R_{2}\right)$ is its $\Delta$-form, a $\Theta$-conservative hypermap with fundamental subgroup $Q$. Hence $\mathcal{Q}$ is regular if and only if its $\Delta$ form $\mathcal{H}$ is $\Theta$-regular. If the $\Theta$-marked fundamental subgroup $Q$ is normal in $\Delta$, we say that $\mathcal{Q}$ is $\Delta$-symmetric. More generally, if $Q$ is normal in some normal subgroup $\Pi$ in $\Delta$, we say that $\mathcal{Q}$ is $\Pi$-symmetric. So a $\Theta$ marked hypermap is $\Delta$-symmetric if and only if it has a regular $\Delta$-form hypermap.

Let us consider the two following $\Theta$-marked hypermaps:

$$
\mathcal{Q}_{b}=\left(\Theta / r Q^{b} ; Q_{\Theta}^{b} x_{1}, \ldots, Q_{\Theta}^{b} x_{m}\right) \quad(\text { the } b \text {-image of } \mathcal{Q})
$$

and

$$
\mathcal{Q}^{b}=\left(\Theta / r Q ; Q_{\Theta} x_{1}^{b^{-1}}, \ldots, Q_{\Theta} x_{m}^{b^{-1}}\right) .
$$

THEOREM $20 . \mathcal{Q}_{b} \cong \mathcal{Q}^{b}$.

Proof. The function $\psi_{b}: \Theta / r Q^{b} \longrightarrow \Theta / r Q=\Theta^{b^{-1}}{ }_{r} Q$ defined by $Q^{b} t \mapsto Q t^{b^{-1}}$ is bijective and for all $t \in \Theta$ and $i \in\{1, \ldots, m\}$ it satisfies

$$
Q^{b} t Q_{\Theta}^{b} x_{i} \psi_{b}=Q^{b} t x_{i} \psi_{b}=Q\left(t x_{i}\right)^{b^{-1}}=Q t^{b^{-1}} x_{i}^{b^{-1}}=Q^{b} t \psi_{b} Q_{\Theta} x_{i}^{b^{-1}}
$$

CoRollary 21. Let $\mathcal{Q}$ be a regular $\Theta$-marked hypermap. Then $\mathcal{Q}$ is $\Delta$-symmetric if and only if $\mathcal{Q} \cong \mathcal{Q}^{b}, \forall b \in T$.

The last theorem of this section will be of great help in the construction of regular $\Theta$-marked hypermaps from given groups.

THEOREM 22. If $G$ is a group generated by $g_{1}, \ldots, g_{m}$ such that the function $\rho: x_{i} \mapsto g_{i}$ extends to an epimorphism from $\Theta$ to $G$, then $\mathcal{Q}=\left(G ; g_{1}, \ldots, g_{m}\right)$ is a regular $\Theta$-marked hypermap. 
Proof. As $G$ acts transitively on itself by right multiplication then $\mathcal{Q}$ is a $\Theta$-marked hypermap. Moreover, the stabiliser of any element of $G$ is trivial. Let $Q=\operatorname{Ker}(\rho)=\rho^{-1}(1)=\operatorname{Stab}_{\Theta}(1)$ under the action of $\Theta$ on $G$ via $\rho$. Then $Q \triangleleft \Theta, G \cong \Theta / Q$ and $\mathcal{Q} \cong\left(\Theta / Q ; Q x_{1}, \ldots, Q x_{m}\right)$. Hence $\mathcal{Q}$ is regular.

\section{6. $\Theta$-slices}

Any hypermap can be topologically constructed by stitching triangular pieces of surface, called flags, following the rule dictated by the monodromy group $[4,17,19,20]$. If the hypermap is orientable, one can use the orientability to reduce the number of surface's pieces by taking larger pieces, called darts, constructed by gluing two adjacent flags along their side labelled 2 (see Fig. 3). This approach can be brought to any $\Theta$-marked hypermap. The resulting building blocks will be called $\Theta$ slices. The "shape" of such a region is not unique, but differs according to the Schreier transversal considered for $\Theta$.

Let $T=\left\{b_{1}=1, \ldots, b_{n}\right\}$ be a Schreier transversal for $\Theta$ in $\Delta$. Associated with $T$ we construct a $\Theta$-slice, in fact a rooted $\Theta$-slice, in the following way. Fix a flag $\omega$, a topological triangle similar to the one displayed in Fig. 1 (left), with their three sides labelled 0, 1, 2 and their corresponding opposite vertices labelled similarly. The side labelled 2, lying on the underlying hypergraph, will be drawn thick while the other two (lying inside of hyperfaces) will be dashed. To get some geometrical meaning we do this on the hyperbolic plane $\mathbb{H}$, modelled by the Poincaré disc. Here a flag is a hyperbolic triangle with internal zero angles. Let $R_{0}, R_{1}$ and $R_{2}$ be the usual reflections on the sides labelled 0,1 and 2 respectively. They generate a group isomorphic to the "triangle" group $\Delta$. Each element $b_{i} \in T$ is a word in $R_{0}, R_{1}, R_{2}$, so $\omega b_{i}$ is an isometric flag in $\mathbb{H}$. The region $\zeta_{\omega}=\cup\{\omega b \mid b \in T\}$, which is connected since $T$ is a Schreier transversal, is our starting rooted $\Theta$-slice (associate to $T$ ) with root flag $\omega$. Fig. 1 (right) displays a rooted $\Theta$-slice, where $\Theta$ is the subgroup $\Delta^{\hat{0} \hat{1}}=\left\langle R_{2},\left(R_{0} R_{1}\right)^{2}\right\rangle^{\Delta}$ of $\Delta$.

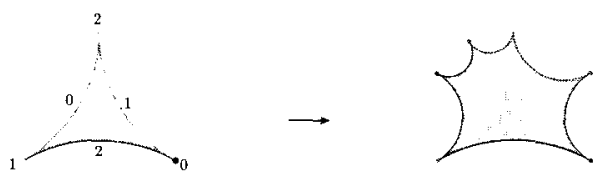

Figure 1: Left: a flag. Right: A rooted $\Delta^{\hat{0} \hat{i}}$-slice, consisting of 4 flags. 
The set of root $\Theta$-flags is $S_{\omega}=\left\{\zeta_{\omega t} \mid t \in \Theta\right\}$ with root-flags $\omega t, t \in \Theta$. The group $\Theta$ acts regularly on $S_{\omega}$ by acting on the root-flags $\omega t$, giving rise to a $\Theta$-tessellation $\mathcal{T}$ of $\mathbb{H}$ by rooted $\Theta$-slices (Fig. 2).

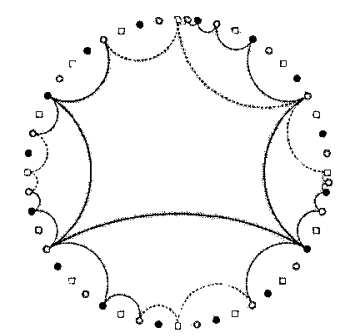

Figure 2: Part of the $\Delta^{0 \hat{0}}$-tessellation in $\mathbb{H}$.

One can choose any other flag $\omega^{\prime}=\omega b(b \in T)$ of $\zeta_{\omega}$ to be a (starting) root-flag; in fact, $T^{\prime}=b^{-1} T$ is also a Schreier transversal for $\Theta$ (see lemma below) and $\zeta_{\omega^{\prime}}^{T^{\prime}}=\zeta_{\omega}^{T}$, so $\zeta_{\omega^{\prime}}^{T^{\prime}}$ represents the same $\Theta$-slice with root-flag $\omega^{\prime}$ (yet associated to $T^{\prime}$ ). Therefore, choosing another flag for root-flag is equivalent to associating the $\Theta$-slice with another Schreier transversal.

Lemma 23. If $T=\left\{1=b_{1}, \ldots, b_{n}\right\}$ is a Schreier transversal for $\Theta$ in $\Delta$, then for $i=1, \ldots, n$ the set $b_{i}^{-1} T=\left\{b_{i}^{-1} b_{1}, \ldots, b_{i}^{-1} b_{n}\right\}$ is still a Schreier transversal for $\Theta$ in $\Delta$.

Proof. The function $\epsilon_{b_{i}}: b_{i}^{-1} T \longrightarrow T, x \mapsto b_{i} x$, is a bijection. Hence $\left|b_{i}^{-1} T\right|=|T|$. On the other hand, for all $b, b^{\prime} \in T, \quad \Theta b_{i}^{-1} b=\Theta b_{i}^{-1} b^{\prime} \Leftrightarrow$ $b_{i}^{-1} \Theta b=b_{i}^{-1} \Theta b^{\prime} \Leftrightarrow \Theta b=\Theta b^{\prime} \Leftrightarrow b=b^{\prime} \Leftrightarrow b_{i}^{-1} b=b_{i}^{-1} b^{\prime}$. Hence $b_{i}^{-1} T$ is a transversal and contains 1 . Let $w \in b_{i}^{-1} T$ such that $w=u v$. Then $b_{i} w=b_{i} u v \in T$, and since $T$ has the Schreier property, $b_{i} u \in T \Leftrightarrow u \in$ $b_{i}^{-1} T$. Hence $b_{i}^{-1} T$ is also a Schreier transversal.

Each $\Theta$-slice $\zeta=\cup\{\omega b \mid b \in T\}$ in $\mathcal{H}$ is a union of $n$ (the index of $\Theta$ in $\Delta$ ) flags, each belonging to a different $\Theta$-orbit. This gives rise to $n$ coloured flags in $\zeta$, inducing $n$ colourings (or roots) to the $\Theta$-slices. Choosing a root colour (or root-flag $\omega$ ) then the action of $\Theta$ on the coloured-flags will fix a root-flag in each $\Theta$-slice giving rise to a set of $n$ coloured rooted $\Theta$-slices $S_{\omega}$. Figure 3 shows $\Theta$-slices for some familiar subgroups $\Theta$ in $\Delta$ with abelian factors, namely, $\Theta=\Delta, \Theta=\Delta^{+}$, $\Theta=\Delta^{\hat{0}}=\left\langle R_{1}, R_{2}\right\rangle^{\Delta}$ and $\Delta^{012}=\left\langle R_{0} R_{1} R_{2}\right\rangle^{\Delta}$. 

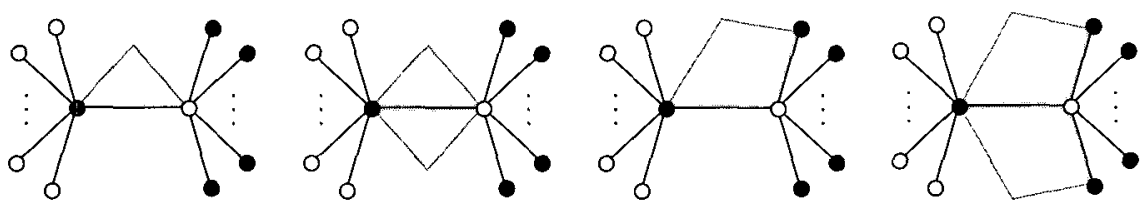

Figure 3: $\Delta$-slice (or flag), $\Delta^{+}$-slice (or dart), $\Delta^{\hat{0}}$-slice and $\Delta^{012}$-slice.

Of course different subgroups $\Theta$ of finite index in $\Delta$ may share the same $\Theta$-slice when taking appropriate Schreier transversals. For example, $\Delta^{0}=\left\langle R_{0}, R_{1} R_{2}\right\rangle^{\Delta}$, of index 2 , may also induce the same $\Delta^{+}$-slice, $\Theta=\Delta^{\hat{0} \hat{2}}=\left\langle R_{1},\left(R_{2} R_{0}\right)^{2}\right\rangle^{\Delta}, \Delta^{+\hat{0}}=\Delta^{+} \cap \Delta^{\hat{0}}$ and $\Delta^{+\hat{2}}=\Delta^{+} \cap \Delta^{\hat{2}}$, all of index 4 , may also induce the same $\Delta^{012}$-slice. For more details on these groups and the regular hypermaps associated to them see [5].

Let $\mathcal{Q}$ be a $\Theta$-marked hypermap with $\Theta$-marked fundamental subgroup $Q$. Being $Q$ a subgroup of $\Theta, Q$ also acts on the rooted $\Theta$-slices. This determines a fundamental region $R \subset \mathbb{H}$, which can be seen as a regular hyperbolic polygon divided into rooted $\Theta$-slices. The orbit space $\mathbb{H} / Q$, which corresponds to the polygon $R$ with their sides pairwise identified, carries a $\Theta$-conservative hypermap $\mathcal{H}$ (also divided into rooted $\Theta$-slices) as an imbedding of the hypergraph determined by the thick lines of the induced tessellation $\mathcal{T}$ on $R$. The induced (topological) $\Theta$-conservative hypermap $\mathcal{H}$ is clearly isomorphic to $\mathcal{Q}$ (in respect to the induced root flags). The choice of another root flag in $\mathcal{H}$ determines a $b$-image of $\mathcal{H}$. When $\mathcal{Q}$ is $\Delta$-symmetric (i.e., its $\Delta$-form is regular), then the choice of a root flag in $\mathcal{H}$ becomes irrelevant. In this case we may discard the root flags and consider $\Theta$-slices instead of rooted $\Theta$-slices.

\section{An example}

Consider the subgroup $\Delta^{\hat{0} \hat{1}}=\left\langle R_{2}, R_{2}^{R_{0}}, R_{2}^{R_{1}}, R_{2}^{R_{0} R_{1}},\left(R_{0} R_{1}\right)^{2}\right\rangle$ with index 4 in $\Delta$. This is one of the seven normal subgroups with index 4 in $\Delta$ [6]. It is isomorphic to a free product $C_{2} * C_{2} * C_{2} * C_{2} *$ $C_{\infty}=\langle A, B, C, D, Z\rangle$. Associated to the Schreier transversal $T=$ $\left\{1, R_{0}, R_{1}, R_{0} R_{1}\right\}$ for $\Delta^{\hat{0} \hat{1}}$ in $\Delta$, we have the rooted $\Delta^{\hat{0} \hat{1}}$-slice shown in Fig. 1 (right).

Let us consider the Klein four group $V_{4}$ and let $\mathcal{Q}$ be the regular $\Delta^{\hat{0} \hat{1}}$-marked hypermap $\left(V_{4}, a, b, c, d, z\right)$, where $V_{4}=\langle a, b\rangle, c=a b$ and $d=z=b$. To construct $\mathcal{Q}$ as a cellular embedding of a hypergraph in some surface $S$, take 4 rooted $\Delta^{\hat{0} \hat{1}}$-slices numerated as $1,2,3$ and 4 . 


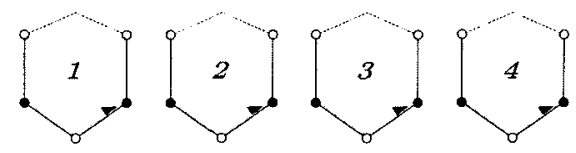

Figure 4: Four rooted $\Delta^{\hat{0} \hat{1}}$-slices.

Since $1 a, 1 a b$ and $1 a b a$ are different $\Delta^{\hat{0} \hat{1}}$-slices, without loss of generality, assign $2=1 a, 3=2 b$ and $4=3 a$. Since $c=\left(r_{0} r_{2}\right)^{2}$ is an involution, we must have these slices joined partially as shown in Fig. 5.

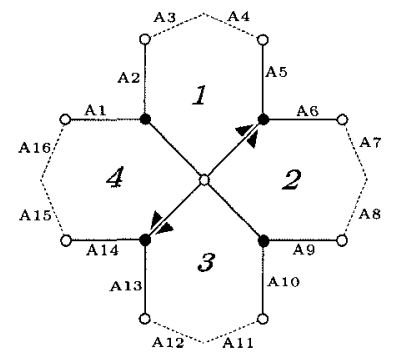

Figure 5: A partial joining of $\Delta^{\hat{0} \hat{1}}$-slices.

Now the equation $c=a b$ applied to the root flags implies $A 5=A 13$ and $A 6=A 14$. The equation $z=b$ on the root flags is equivalent to $r_{1}^{r_{0}}=r_{2}^{r_{0}} r_{1}$ on root flags, and this implies $A 3=A 15, A 8=A 12$, $A 16=A 4$ and $A 11=A 7$. Similarly, $d=z$ on the root flags is equivalent to $r_{1} r_{0}=r_{0} r_{1} r_{2}$ and this implies $A 1=A 2$ and $A 9=A 10$. The final picture is shown in Fig. 6.

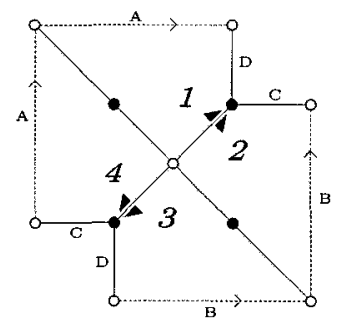

Figure 6: The cellular hypergraph embedding of $\mathcal{Q}$.

The $\Delta$-form of $\mathcal{Q}$ is $\Delta^{\hat{0} \hat{1}}$-regular. 


\section{8. $\Theta$-regularity as a regular covering}

The trivial $\Theta$-hypermap $\mathcal{T}_{\Theta}$, that is, the regular hypermap with fundamental subgroup $\Theta$, is the smallest $\Theta$-conservative hypermap. Its flag's set is the quotient $\Delta / \Theta$ and hence the number of flags of $\mathcal{T}_{\Theta}$ is the index of $\Theta$ in $\Delta$. If a hypermap $\mathcal{H}$ is $\Theta$-conservative, its fundamental subgroup $H$ is a subgroup of $\Theta$ and so we have a covering $\rho: \mathcal{H} \longrightarrow \mathcal{T}_{\Theta}$ given by $\rho: \Delta / r H \rightarrow \Delta / \Theta, H d \mapsto \Theta d$. The size of $\mathcal{T}_{\Theta}$ can be seen as the number of $\Theta$-orbits in $\mathcal{H}$. The covering $\rho$ is regular if $H \triangleleft \Theta$, that is, if $\mathcal{H}$ is $\Theta$-regular, in which case the covering group is given by $\Theta / H$.

Let $T=\left\{b_{1}=1, b_{2}, \ldots, b_{n}\right\}$ be a transversal for $\Theta$ in $\Delta$. Being $\mathcal{H}$ $\Theta$-conservative then each $\Theta$-orbit $F_{b_{i}}^{\Theta}=\left\{H b_{i} t \mid t \in \Theta\right\}$ in $\mathcal{H}$ is a "fiber" $\left(\Theta b_{i}\right) \rho^{-1} ;$ in fact,

$$
\begin{aligned}
\left(\Theta b_{i}\right) \rho^{-1} & =\left\{H d \in \Delta / r H \mid \Theta d=\Theta b_{i}\right\} \\
& =\left\{H t b_{i} \in \Delta / r H \mid t \in \Theta\right\} \\
& =\left\{H b_{i} t^{\prime} \in \Delta / r H \mid t^{\prime}=b_{i}^{-1} t b_{i}=t^{b_{i}} \in \Theta\right\} \\
& =F_{b_{i}}^{\Theta}
\end{aligned}
$$

The function $\phi:(\Theta d) \rho^{-1} \longrightarrow \Theta \rho^{-1}=\Theta / H, H g \mapsto H g d^{-1}$, is a bijection from any fiber $(\Theta d) \rho^{-1}$ to the "main" fiber $\Theta \rho^{-1}=\Theta / H$. This confirms an earlier result saying that any two $\Theta$-orbits have the same length.

\section{9. $\Theta$-type and characteristic of a $\Theta$-regular hypermap}

Let $\mathcal{H}$ be a $\Theta$-regular hypermap. The $\Theta$-automorphism group $\operatorname{Aut}^{\ominus}(\mathcal{H})$ acts regularly on any $\Theta$-orbit. For each hypervertex $v$ of $\mathcal{H}$, denote by $F_{v}$ the set of flags incident with $v$. The " $\Theta$-stabiliser"

$$
E_{F_{v}}^{\Theta}=\operatorname{Stab}_{\operatorname{Aut}_{(\mathcal{H})}^{\Theta}}\left(F_{v}\right)
$$

is a subgroup of $\left\langle r_{1}, r_{2}\right\rangle \subset \operatorname{Aut}^{\ominus}(\mathcal{H})$ (which is cyclic or dihedral). Since $E_{F_{v}}^{\Theta}$ is a subgroup of $\operatorname{Aut}^{\Theta}(\mathcal{H})$ it acts regularly on the subset of flags $\omega \Theta \cap$ $F_{v}$ of each $\Theta$-orbit $\omega \Theta$ meeting $v$. Then $\left|\omega \Theta \cap F_{v}\right|=\left|E_{F_{v}}^{\Theta}\right|$ and hence part (1) of the next theorem is established. Since automorphisms preserve incidence, any automorphism $\psi$ can be seen as a function that sends $i$-hypercells (0-hypercells $=$ hypervertices, 1-hypercells=hyperedges and 2 -hypercells=hyperfaces) to $i$-hypercells. Moreover, incidence shows 
that for any hypercell $c, F_{\mathrm{c}} \psi=F_{c \psi}$. So if $\varphi$ is a $\Theta$-automorphism then for any flag $\omega, \omega \Theta \varphi=\omega \Theta$ and so, for any hypervertex $v$,

$$
\left(\omega \Theta \cap F_{v}\right) \varphi=\omega \Theta \cap F_{v \varphi} .
$$

This helps to finish the proof of the following theorem.

Theorem 24. Let $\mathcal{H}$ be a $\Theta$-regular hypermap.

(1) If two $\Theta$-orbits meet in one hypervertex $v$ (resp. hyperedge, hyperface), then they both meet $v$ with $\left|E_{F_{v}}^{\ominus}\right|$ flags.

(2) Let $\omega \Theta$ be a $\Theta$-orbit meeting two different vertices $u$ and $v$. If $\omega \Theta$ meets $u$ with $k$ flags, then $\omega \Theta$ also meets $v$ with $k$ flags.

(3) If two $\Theta$-orbits meet in one hypervertex (resp. hyperedge, hyperface), then they will meet at exactly the same hypervertices (resp. hyperedges, hyperfaces).

Proof. (2) The transitivity of $\operatorname{Aut}^{\Theta}(\mathcal{H})$ on $\Theta$-orbits implies that there is $\phi \in \operatorname{Aut}^{\Theta}(\mathcal{H})$ such that $u \phi=v$. Then $\left(\omega \Theta \cap F_{u}\right) \phi=\omega \Theta \cap F_{v}$ and so $\left|\omega \Theta \cap F_{v}\right|=\left|\omega \Theta \cap F_{u}\right|=k$.

(3) Let $\omega_{1} \Theta, \omega_{2} \Theta$ be two $\Theta$-orbits meeting the hypervertex $u$. Without loss of generality we may suppose that $\omega_{1}, \omega_{2} \in F_{u}$. If $\omega_{1} \Theta$ meets another hypervertex $v$, then the transitivity of $\operatorname{Aut}^{\ominus}(\mathcal{H})$ on $\Theta$-orbits implies that there is $\phi \in \operatorname{Aut}^{\Theta}(\mathcal{H})$ such that $u \phi=v$. Then $\left(\omega_{2} \Theta \cap F_{u}\right) \phi=\omega_{2} \Theta \cap F_{v} \neq$ $\emptyset$, that is, $\omega_{2} \Theta$ also meets $v$.

Two distinct $\Theta$-orbits may meet different numbers of hypervertices (resp. hyperedges, hyperfaces). However, in a $\Theta$-regular hypermap $\Theta-$ orbits lie inside $\operatorname{Aut}(\mathcal{H})$-orbits, and so, if two distinct $\Theta$-orbits lie inside the same $\operatorname{Aut}(\mathcal{H})$-orbit then the following result says that despite they may not meet the same hypervertices they will meet the same number of hypervertices.

ThEOREM 25. Let $\mathcal{H}$ be a $\Theta$-regular hypermap and $\omega_{1}$ e $\omega_{2}$ be two flags in the same $\operatorname{Aut}(\mathcal{H})$-orbit. Then the $\Theta$-orbits $\omega_{1} \Theta$ and $\omega_{2} \Theta$ meet the same number of hypervertices (resp. hyperedges, hyperfaces) with the same number of flags.

Proof. This is a consequence of $\omega_{1} \Theta \psi=\omega_{1} \psi \Theta$, for any automorphism $\psi \in \operatorname{Aut}(\mathcal{H})$. In fact, since $\omega_{1}$ and $\omega_{2}$ belong to an $\operatorname{Aut}(\mathcal{H})$-orbit, there is $\psi \in \operatorname{Aut}(\mathcal{H})$ such that $\omega_{2}=\omega_{1} \psi$. If $\omega_{1} \Theta$ meets the $k$ hypervertices $v_{1}, \ldots, v_{k}$, so $v_{1}, \ldots, v_{k}$ are all the hypervertices such that $\omega_{1} \Theta \cap F_{v_{i}} \neq \emptyset$, for any $i=1, \ldots, k$, then also $\left(\omega_{1} \Theta \cap F_{u_{i}}\right) \psi=\omega_{2} \Theta \cap F_{u_{i} \psi} \neq \emptyset$. Since $\psi$ is invertible, $u_{1} \psi, \ldots, u_{k} \psi$ are all the hypervertices that $\omega_{2} \Theta$ meets. By 
Part (2) of Theorem 24, the sets $\omega_{1} \Theta \cap F_{u_{i}}, i=1, \ldots, k$, all have the same cardinality, thus so do the sets $\omega_{2} \Theta \cap F_{u_{i} \psi}$ for $i=1, \ldots, k$.

Consider the regular covering $\rho: \mathcal{H} \longrightarrow \mathcal{T}_{\Theta}$. As we saw in $\S 8$, each $\Theta$-orbit is a fiber $\left(\Theta b_{i}\right) \rho^{-1}$. Let $v$ be a hypervertex of $\mathcal{T}_{\Theta}$ and $w$ a flag in $v$. The valency of $v$ divides the valency of each hypervertex $v_{i} \in\{v\} \rho^{-1}$ in $\mathcal{H}$ that projects over $v$. The $\Theta$-orbit $\{w\} \rho^{-1}$ meets every hypervertex of $\{v\} \rho^{-1}$, hence, by Theorem 24 , all the hypervertices in $\{v\} \rho^{-1}$ share the same valency. Similarly, all the hyperedges (resp. hyperfaces) in $\mathcal{H}$ that cover an hyperedge (resp. hyperface) of $\mathcal{T}_{\Theta}$ share the same valency.

Let $\left\{v_{1}, \ldots, v_{q_{v}}\right\},\left\{e_{1}, \ldots, e_{q_{e}}\right\}$ and $\left\{f_{1}, \ldots, f_{q_{f}}\right\}$ be the hypervertices, hyperedges and hyperfaces (respectively) of the trivial $\Theta$-hypermap $\mathcal{T}_{\Theta}$ and $(k ; l ; m)$ be the type of $\mathcal{T}_{\Theta}$; so $q_{v}, q_{e}$ and $q_{f}$ are the number of hypervertices, hyperedges and hyperfaces of $\mathcal{T}_{\ominus}$, respectively. Let $\mathcal{V}_{i}=$ $\left\{v_{i}\right\} \rho^{-1}, \mathcal{E}_{i}=\left\{e_{i}\right\} \rho^{-1}$ and $\mathcal{F}_{i}=\left\{f_{i}\right\} \rho^{-1}$ be the sets of hypervertices, hyperedges and hyperfaces of $\mathcal{H}$ projecting via $\rho$ to $v_{i}, e_{i}$ and $f_{i}$ respectively. These have common valencies respectively $k_{i}, l_{i}$ and $m_{i}$. Notice that $k$ divides each $k_{i}\left(i=1, \ldots, q_{v}\right), l$ divides each $l_{i}\left(i=1, \ldots, q_{e}\right)$ and $m$ divides each $m_{i}\left(i=1, \ldots, q_{f}\right)$. The following sequence

$$
\left(k_{1}, \ldots, k_{q_{v}} ; l_{1}, \ldots, l_{q_{e}} ; m_{1}, \ldots, m_{q_{f}}\right)
$$

will be called the $\Theta$-type of $\mathcal{H}$. The $q_{v}$ hypervertices (resp. $q_{e}$ hyperedges and $q_{f}$ hyperfaces) of $\mathcal{T}_{\Theta}$ give rise to $q_{v} 0$-colours (or vertex-colours) among the hypervertices of $\mathcal{H}$ (resp. $q_{e} 1$-colours and $q_{f}$ 2-colours). The underlying hypergraph $\mathcal{G}$ of $\mathcal{H}$ is then " $\left(q_{v}, q_{e}\right)$-coloured", that is, as a bipartite graph it is a $\left(q_{v}+q_{e}\right)$-vertex coloured graph.

\subsection{Characteristic of a $\Theta$-regular hypermap without bound- ary}

Denote by $\mathcal{V}=\cup_{i=1}^{q_{v}} \mathcal{V}_{i}, \mathcal{E}=\cup_{i=1}^{q_{e}} \mathcal{E}_{i}$ and $\mathcal{F}=\cup_{i=1}^{q_{f}} \mathcal{F}_{i}$ the sets of hypervertices, hyperedges and hyperfaces of $\mathcal{H}$. Then $\sum_{i=1}^{q_{v}}\left|\mathcal{V}_{i}\right|=|\mathcal{V}|$, $\sum_{i=1}^{q_{e}}\left|\mathcal{E}_{i}\right|=|\mathcal{E}|$ and $\sum_{i=1}^{q_{f}}\left|\mathcal{F}_{i}\right|=|\mathcal{F}|$. As all the hypervertices in $\mathcal{V}_{i}$ have the same valency $k_{i}$, and $\mathcal{H}$ has no boundary, then we have $2 k_{i}\left|\mathcal{V}_{i}\right|$ flags lying on $\mathcal{V}_{i}$. On the other hand, the hypervertices of $\mathcal{V}_{i}$ project over $v_{i}$ and around $v_{i}$ we have $\mu_{v} k$ flags, where $\mu_{v}=1$ if $v$ is on the boundary of $\mathcal{T}_{\Theta}$ and $\mu_{v}=2$ if not. Then the number of flags lying around the vertices of $\mathcal{V}_{i}$ must also be given by $\mu_{v} k|\Theta: H|$; that is,

$$
2 k_{i}\left|\mathcal{V}_{i}\right|=\mu_{v} k|\Theta: H| \text {. }
$$


Analogously we define the numbers $\mu_{e}$ for hyperedges and $\mu_{f}$ for hyperfaces, with similar formulas

$$
\begin{aligned}
2 l_{i}\left|\mathcal{E}_{i}\right| & =\mu_{e} l|\Theta: H|, \\
2 m_{i}\left|\mathcal{F}_{i}\right| & =\mu_{f} m|\Theta: H| .
\end{aligned}
$$

As $|F|=n|\Theta: H|$ then the characteristic $\chi$ of $\mathcal{H}$ is given by

$$
\begin{aligned}
\chi(\mathcal{H}) & =|\mathcal{V}|+|\mathcal{E}|+|\mathcal{F}|-\frac{|F|}{2} \\
& =\sum_{i=1}^{q_{v}}\left|\mathcal{V}_{i}\right|+\sum_{i=1}^{q_{e}}\left|\mathcal{E}_{i}\right|+\sum_{i=1}^{q_{f}}\left|\mathcal{F}_{i}\right|-\frac{n|\Theta: H|}{2} \\
& =\sum_{i=1}^{q_{v}} \frac{\mu_{v} k|\Theta: H|}{2 k_{i}}+\sum_{i=1}^{q_{e}} \frac{\mu_{e} l|\Theta: H|}{2 l_{i}}+\sum_{i=1}^{q_{f}} \frac{\mu_{f} m|\Theta: H|}{2 m_{i}}-\frac{n|\Theta: H|}{2} \\
& =|\Theta: H|\left(\sum_{i=1}^{q_{v}} \frac{\mu_{v} k}{2 k_{i}}+\sum_{i=1}^{q_{e}} \frac{\mu_{e} l}{2 l_{i}}+\sum_{i=1}^{q_{f}} \frac{\mu_{f} m}{2 m_{i}}-\frac{n}{2}\right) .
\end{aligned}
$$

For $\Theta$-regular hypermaps of negative characteristic $N=-\chi>0$, this formula permits the evaluation of an upper bounding for $\left|\mathrm{Aut}^{\Theta}(\mathcal{H})\right|=$ $|\Theta: H|$ for each ordering of $\left\{k_{1}, \ldots, k_{q_{v}}, l_{1}, \ldots, l_{q_{e}}, m_{1}, \ldots, m_{q_{f}}\right\}$. Choosing the highest upper bounding on all possible orderings (the number of orderings is finite), we get the following kind of "Riemann-Hurwitz" bound:

Theorem 26. For each $N>0$, there is $\Sigma_{\ominus}<\frac{n}{2}$ such that

for any $\Theta$-regular hypermap $\mathcal{H}$.

$$
\left|A u t^{\Theta}(\mathcal{H})\right| \leq \frac{N}{\frac{n}{2}-\Sigma_{\Theta}}
$$

For example, for $\Theta=\Delta$ the bound is $84 N$, for $\Theta=\Delta^{+}$the bound is $42 N$ (the Hurwitz bound $84(g-1)$ as it is known, where $g$ is the genus), for $\Theta=\Delta^{0}$ the bound is $24 N$ and for $\Theta=\Delta^{012}$ the bound is $6 N$. Since any $\Theta$-regular hypermap is fully determined by its $\Theta$-automorphism group we have,

Corollary 27. For each $\Theta$ and for each $N>0$ the number of $\Theta$ regular hypermaps of negative characteristic $N$ is finite.

In other words, given a regular hypermap $\mathcal{T}$, the number of hypermaps $\mathcal{H}$ of given negative characteristic $N>0$ that regularly covers $\mathcal{T}$ is finite.

\section{Restrictly regular hypermaps}

We say that a hypermap $\mathcal{H}$ is restrictly regular if $\mathcal{H}$ is $\Theta$-regular for some normal subgroup $\Theta$ with finite index in $\Delta$. The restricted rank $r$ 
of a restrictly regular hypermap is the index $r$ of the greatest normal subgroup $\Theta$ in $\Delta$ such that $\mathcal{H}$ is $\Theta$-regular. Restricted rank 1 means that $\mathcal{H}$ is $\Delta$-regular, or simply regular. Let the restricted co-rank of a hypermap $\mathcal{H}$ to be the quotient $|F| / r$; this is the $\Theta$-orbit's length of a $\Theta$-regular hypermap of restricted rank $r=|\Delta: \Theta|$. As the length of a $\Theta$-orbit of a $\Theta$-regular hypermap $\mathcal{H}$ must divide $|\operatorname{Aut}(\mathcal{H})|$, which is the length of an $\operatorname{Aut}(\mathcal{H})$-orbit, we have:

Lemma 28. The restricted co-rank of a restrictly regular hypermap $\mathcal{H}$ must be a divisor of $|A u t(\mathcal{H})|$.

For example, if $\mathcal{H}$ is not regular and has trivial automorphism group then $\mathcal{H}$ cannot be restrictly regular. In fact, if $\mathcal{H}$ is $\Theta$-regular for some $\Theta \triangleleft \Delta$, since $\operatorname{Aut}^{\Theta}(\mathcal{H})<\operatorname{Aut}(\mathcal{H})$ then $|\Theta / H|=\left|F^{\Theta}\right|=\left|\operatorname{Aut}^{\Theta}(\mathcal{H})\right|=1$, where $H$ is the fundamental subgroup of $\mathcal{H}$. So $H=\Theta$ and $\mathcal{H}$ is regular, which is against our assumption. Examples providing larger automorphism groups is given by the following theorem, where hyperfaces can be replaced by hypervertices or hyperedges.

THEOREM 29. Let $\mathcal{H}$ be a hypermap with a hyperface $A$ of valency $m$ and another $B$ of valency $n$. If $m$ and $n$ are coprimes, and the rotation one step about $A$ or $B$ is not an automorphism of $\mathcal{H}$, then $\mathcal{H}$ cannot be restrictly regular.

Proof. Since $\mathcal{H}$ is not regular the automorphism group $\operatorname{Aut}(\mathcal{H})$ acts with at least 2 orbits in the set of flags $F$ of $\mathcal{H}$. Suppose that $\mathcal{H}$ is $\Theta$-regular for some normal subgroup $\Theta$ of finite index in $\Delta$. Let $H$ be the fundamental subgroup of $\mathcal{H}$ and let $\beta=H \in F=\Delta / r H$ be a flag in $A$. Since $\mathcal{H}$ is $\Theta$-conservative, $H \subset \Theta$, and since $\beta\left(r_{0} r_{1}\right)^{m}=\beta$, that is, $H H_{\Delta}\left(R_{0} R_{1}\right)^{m}=H$, then $\left(R_{0} R_{1}\right)^{m} \in H$, and so, $\left(R_{0} R_{1}\right)^{m} \in \Theta$. If $\gamma=H d \in F$ is a flag in $B$ then $\gamma\left(r_{0} r_{1}\right)^{n}=\gamma$, which implies that $\left(R_{0} R_{1}\right)^{n} \in H^{d} \subset \Theta$. Now $\operatorname{gcd}(m, n)=1$, so there are integers $p, q$ such that $p m+q n=1$, and so, $R_{0} R_{1}=\left(R_{0} R_{1}\right)^{p m}\left(R_{0} R_{1}\right)^{q n} \in \Theta$. Now the $\Theta$-regularity of $\mathcal{H}$ implies that Aut $^{{ }^{\theta}}(\mathcal{H})$ acts regularly on both $\Theta$-orbits $\beta \Theta$ and $\gamma \Theta$. Since $\Theta$ contains $R_{0} R_{1}$, this means that the rotations one step on both hyperfaces $A$ and $B$ are automorphisms of $\mathcal{H}$, which is against our hypothesis.

Corollary 30. If $\mathcal{M}$ is a regular map of type $\{p, q\}$ with $\operatorname{gcd}(2 p, q)=$ 1 , then the truncated map $T \mathcal{M}$ is not restrictly regular.

Proof. $T \mathcal{M}$ has two type of faces, the face-faces (faces originating from the original faces) of valency $2 p$ and the vertex-faces (faces originating from the vertices) of valency $q$. The rotation one step about a 
face-face cannot be an automorphism of $T \mathcal{M}$ since it takes a face-face to a vertex-face with different valency.

It is therefore reasonable to ask when a given hypermap $\mathcal{H}$ is, or is not, restrictly regular. Let $\mathcal{H}$ be a hypermap with fundamental subgroup $H$. The normaliser $N_{\Delta}(H)$ gives rise to a not necessarily regular hypermap $\mathcal{N}$, call it the normaliser of $\mathcal{H}$. The hypermap $\mathcal{H}$ regularly covers $\mathcal{N}$, with covering transformation group $N_{\Delta}(H) / H \cong \operatorname{Aut}(\mathcal{H})$. Denote by $\underline{\mathcal{H}}$ the regular hypermap with fundamental subgroup $\Phi=\left(N_{\Delta}(H)\right)$. This group $\Phi$, which we will call the regularity-subgroup of $\mathcal{H}$, is the greatest normal subgroup $\Theta$ in $\Delta$ such that $\mathcal{H}$ has the possibility to be $\Theta$-regular. If $\mathcal{H}$ is regular, then $\Phi=\Delta$ and consequently $\Delta / \Phi$ is trivial. Note that $\Phi$ is a subgroup of $N_{\Delta}(H)$, so if $H<\Phi$ then $H \triangleleft \Phi$ and $\Phi / H=\operatorname{Aut}^{\Phi}(\mathcal{H})<\operatorname{Aut}(\mathcal{H})$.

THEOREM 31. $\mathcal{H}$ is restrictly regular if and only if the regularitysubgroup contains the fundamental subgroup . Moreover, if $\mathcal{H}$ is restrictly regular then the regularity-subgroup $\Phi$ is the greatest normal subgroup $\Theta$ with finite index in $\Delta$ such that $\mathcal{H}$ is $\Theta$-regular, and hence, $\mathcal{H}$ has restricted rank $|\Delta: \Phi|$.

Proof. If $\mathcal{H}$ is $\Theta$-regular for some normal subgroup $\Theta$ (with finite index) in $\Delta$, then $H \subset \Theta \subset N_{\Delta}(H)$. As $\Theta \triangleleft \Delta$ then $\Theta \subset\left(N_{\Delta}(H)\right)_{\Delta}$ and hence $H \subset\left(N_{\Delta}(H)\right)_{\Delta}$.

Conversely if $H \subset\left(N_{\Delta}(H)\right)_{\Delta}$, since $H \triangleleft N_{\Delta}(H)$ and $\left(N_{\Delta}(H)\right)_{\Delta}<$ $N_{\Delta}(H)$, then $H \triangleleft\left(N_{\Delta}(H)\right)_{\Delta}$ and $\mathcal{H}$ is $\Theta$-regular for $\Theta=\left(N_{\Delta}(H)\right)_{\Delta}$, which is a normal subgroup of $\Delta$ with finite index.

Not every hypermap is restrictly regular, in other words, not every hypermap regularly covers a regular hypermap. As mentioned earlier, if $\mathcal{H}$ has trivial automorphism group then $N_{\Delta}(H)=H$ and so, $\Phi=$ $\left(N_{\Delta}(H)\right)_{\Delta}=H_{\Delta}$. If $H \neq \Delta$, then $H$ strictly contains $H_{\Delta}$ and $\mathcal{H}$ is not restrictly regular.

Of the 14 automorphism types of edge-transitive maps classified by Graver and Watkins [15], see also [27], 11 correspond to restrictly regular maps, namely: type 1 corresponds to $\Delta$-regularity; types $2,2^{*}, 2^{\mathrm{P}}, 2 \mathrm{ex}$, $2^{*}$ ex and $2^{\mathrm{P}}$ correspond to $\Delta^{\hat{0}_{-}}, \Delta^{\hat{2}_{-}}, \Delta^{1_{-},} \Delta^{2}{ }_{-}, \Delta^{0}-$ and $\Delta^{+}$-regularity of restricted rank 2 ; type 3 corresponds to $\Delta^{\hat{0} \hat{2}}$-regularity of restricted rank 4 ; types $5,5^{*}$ and $5^{\mathrm{P}}$ correspond to $\Delta^{+\hat{0}_{-}}, \Delta^{+\hat{2}_{-}}$and $\Delta^{012}$-regularity of restricted rank 4 . 
The Buckminsterfullerene (or buckyball), the prototypical member $C_{60}$ of a family of highly symmetrical carbon-cage molecules whose discovery has led to the 1996 Nobel Prize in Chemistry, can be modelled as a truncated Icosahedron. As the Icosahedron is a regular map of type $\{3,5\}$ and $\operatorname{gcd}(3,10)=1$, Theorem 30 says that the buckyball is not restrictly regular. This apparent contradiction to its highly symmetrical shape has an explanation. We have been representing a hypermap as a bipartite map. However, traditionally a hypermap $\mathcal{H}$ has been defined as a cellular imbedding of a 3 -valent graph $\mathcal{G}$ whose faces can be 3 -coloured in such a way that they all meet at each vertex. The vertices of $\mathcal{G}$ is the set of flags and the 3 coloured faces make up the hypervertices, hyperedges and the hyperfaces of $\mathcal{H}$. The bipartite map representation arises when the 0 - and 1 - faces (the coloured faces that represent the hypervertices and hyperedges, respectively) shrink to points, the vertices of the bipartite map. If $\mathcal{H}$ is a map, we can shrink the 1-faces, "rectangles" like faces, to edges. The buckyball, or the truncated icosahedron, seen in this way represents the map Icosahedron as an imbedding of a 3-valent graph whose 1-faces were shrinked to edges. In this view, the buckyball is a regular hypermap.

The cellular graph imbedding (or map) appearing in Picture 10 of page 405 of Burnside monograph [11], is clearly not regular, but, unlike the buckyball, it is restrictly regular. Redrawing the map in the Riemann sphere, or inserting $\infty$ to form a 1-compactification of the plane, the Burnside's map becomes the picture shown in Figure 7(a).

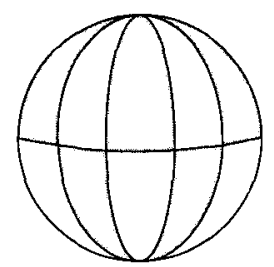

(a)

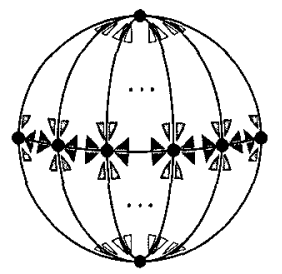

(b)

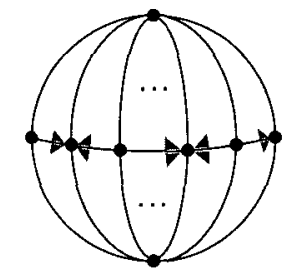

(c)

Figure 7: A sphere with $n$ (even) sections with an equatorial rim.

Burnside considered only the darker regions to represent a dihedral group $D_{\frac{n}{2}}$. Let us assume $n$ either even or odd and let $\mathcal{M}$ be the map. The automorphism group of $\mathcal{M}$ is a direct product $D_{n} \times C_{2}$ that acts with 3 orbits, the white, dark and grey flags (Fig. 7(b)). Hence the restricted rank $r$ shall be greater than 3. If $n$ is odd, we have two vertices whose valency are coprime. Since the rotation one step about 
an equatorial vertex is not an automorphism of $\mathcal{M}$, by Theorem 29 , $\mathcal{M}$ is not restrictly regular. If $n$ is even, let us show that $\mathcal{M}$ is restrictly regular with restricted rank 6 . The number of flags is $12 n$ and each $\operatorname{Aut}(\mathcal{M})$-orbit has $4 n=|\operatorname{Aut}(\mathcal{M})|$ flags. By lemma 28 , the restricted co-rank $s$ must divide properly $4 n$, i.e., $s \neq 4 n$; the highest possible value is obtained when $s=2 n$, giving a possible value of 6 for its rank $r$. The normal closure $\Theta=\left\langle R_{2},\left(R_{0} R_{1}\right)^{3}\right\rangle^{\Delta}$ has index 6 in $\Delta$ and since $R_{2}, R_{2}^{R_{0}},\left(R_{0} R_{2}\right)^{2}=R_{2}^{R_{0}} R_{2}, R_{2}^{R_{1}},\left(R_{1} R_{2}\right)^{2}=R_{2}^{R_{1}} R_{2},\left(R_{1} R_{2}\right)^{4} \in \Theta$, it is not difficult to see that $\mathcal{M}$ is $\Theta$-conservative. As a $\Theta$-orbit lies inside an $\operatorname{Aut}(\mathcal{M})$-orbit (Fig. $7(\mathrm{c})), \operatorname{Aut}^{\Theta}(\mathcal{M})$ acts transitively on a $\Theta$-orbit, so $\mathcal{M}$ is $\Theta$-regular. Hence $\mathcal{M}$ is restrictly regular with restricted rank 6 . Note that by Riedmeister-Schreier's Rewriting Process, $\Theta=\langle A, B, C, D, E, F, Z\rangle \cong C_{2} * C_{2} * C_{2} * C_{2} * C_{2} * C_{2} * C_{\infty}$, where $A=R_{2}, B=R_{2}^{R_{0}}, C=R_{2}^{R_{0} R_{1}}, D=R_{2}^{R_{0} R_{1} R_{0}}, E=R_{2}^{R_{1}}, F=R_{2}^{R_{1} R_{2}}$ and $Z=\left(R_{0} R_{1}\right)^{3}$. The regular $\Theta$-marked map induced by $\mathcal{H}$ is $\mathcal{Q}=$ $\left(D_{\frac{n}{2}} \times C_{2} ; a, a, c, d, c, c, 1\right)$, where $D_{\frac{n}{2}}=\left\langle c, d \mid c^{2}=d^{2}=(c d)^{\frac{n}{2}}=1\right\rangle$ and $C_{2}=\langle a\rangle$.

Meanwhile, we may see the dark and white regions as dark and white flags. In this way Fig. 7(a) is a regular map on the sphere with two vertices (the poles), without the equatorial rim. Burnside's view correspond to the oriented version of this, a regular oriented map with dihedral automorphism group.

\section{References}

[1] D. Archdeacon, J. Širán̆, and M. Škoviera, Self-dual regular maps from medial graphs, Acta Math. Univ. Comenian. 61 (1992), no. 1, 57-64.

[2] G. V. Belyì, On Galois extensions of a maximal cyclotomic field, Math. USSRIzv. 14 (1980), no. 2, 247-256.

[3] H. R. Brahana, Regular maps on an anchor ring, Amer. J. Math. 48 (1926), no. 4, 225-240.

[4] A. Breda d'Azevedo, The Reflexible Hypermaps of Characteristic -2, Math. Slovaca 47 (1997), no. 2, 131-153.

[5] A. Breda d'Azevedo and G. Jones, Double coverings and reflexible abelian hypermaps, Beiträge Algebra Geom. 41 (2000), no. 2, 371-389.

[6] A. Breda d'Azevedo, G. Jones, R. Nedela, and M. Škoviera, Chirality groups of maps and hypermaps, Submitted.

[7] A. Breda d'Azevedo and R. Nedela, Chiral hypermaps of small genus, Beiträge Algebra Geom. 44 (2003), no. 1, 127-143.

[8] Chiral hypermaps with few hyperfaces, Math. Slovaca 53 (2003), no. 2, $107-128$.

[9] Join and Intersection of hypermaps, Acta Univ. M. Belii Math. No. 9 (2001), 13-28. 
[10] R. P. Bryant and D. Singerman, Foundations of the theory of maps on surfaces with boundary, Quart. J. Math. Oxford Ser. (2) 36 (1985), no. 141, 17-41.

[11] W. Burnside, Theory of groups of finite order (reprinted, 2nd edition), Dover, New York, 1955.

[12] D. Corn and D. Singerman, Regular hypermaps, European J. Combin. 9 (1988), no. $4,337-351$.

[13] W. Dyck, Gruppentheoretischen Studien, Math. Ann. 20 (1882), no. 1, 1-44.

[14] A. Errera, Sur les Polyèdres Réguliers de l'Analysis Situs, Bruxelles, 1922.

[15] J. Graver and M. E. Watkins, Locally finite, planar, edge-transitive graphs, Memoirs Am. Math. Soc. 126 (1997), no. 601, vi+75 pp.

[16] A. Grothendieck, Esquisse d'un programme (1984), In: Schneps L., Lochak P, eds. "Geometrie Galois Action Vol 1: Around Grothendieck's esquisse d'un Programme", London Math. Soc. Lecture Notes Series,242 Cambridge Univ. Press (1997), 243-284.

[17] G. A. Jones, Graph imbeddings, groups and Riemann surfaces, In: Algebraic Methods in Graph Theory, Szeged 1978 (L. Lovász, V. T. Sód, eds), NorthHolland, Amsterdam (1981), 297-311.

[18] _ , Maps on surfaces and Galois groups, Math. Slovaca 47 (1997), no. 1, $1-33$.

[19] G. A. Jones and D. Singerman, Theory of maps on orientable surfaces, Proc. London Math. Soc. 37 (1978), no. 2, 273-307.

[20] _ Maps, hypermaps and triangle groups, Proc. London Math. Soc., Lecture Notes Ser. 200 (1994), 115-145.

[21] _ Bely 28 (1996), no, 6, 561-590.

[22] G. A. Jones and J. S. Thornton, Operations on maps, and outer automorphisms, J. Combin. Theory Ser. B 35 (1983), no. 2, 93-103.

[23] R. C. Lyndon and P. E. Schupp, Combinatorial Group Theory, Springer, Berlin, 1977.

[24] R. Nedela, Regular maps - combinatorial objects relating different fields of mathematics, J. Korean Math. Soc. 38 (2001), no. 5, 1069-1105.

[25] D. Singerman, Automorphisms of maps, permutations groups and Riemann surfaces, Bull. Amer. Math. Soc. 8 (1976), no. 1, 65-68.

[26] J. Siráñ, Triangle group representations and constructions of regular maps, Proc. London Math. Soc. (3) 82 (2001), no. 3, 513-532.

[27] J. Širáñ, T. W. Tucker, and M. E. Watkins, Realizing finite edge-transitive orientable maps, J. Graph Theory 37 (2001), no. 1, 1-34.

[28] S. Wilson, New Techniques for the Construction of Regular Maps, University of Washington, 1976.

[29] H. Zassenhaus, Theory of Groups, Chelsea Publishing Company, New York, 1958.

Department of Mathematics

University of Aveiro

Aveiro, Portugal

E-mail: breda@mat.ua.pt 